\title{
Beam Profiling of Dental Light Curing Units Using Different Camera-Based Systems
}

\author{
Mateus Garcia Rocha ${ }^{1}$ Dayane Oliveira ${ }^{1}$ Christopher Felix ${ }^{2}$ Jean-François Roulet ${ }^{1}$ \\ Mário Alexandre Coelho Sinhoreti ${ }^{3}$ Américo Bortolazzo Correr ${ }^{3}$ \\ ${ }^{1}$ Operative Dentistry Division, Department of Restorative Dental \\ Sciences, College of Dentistry, University of Florida, Florida, \\ United States \\ 2Bluelight Analytics Inc., Nova Scotia, Canada \\ ${ }^{3}$ Dental Biomaterials Division, Department of Restorative Dentistry,

\begin{abstract}
Address for correspondence Mateus Garcia Rocha, DDS, MSc, PhD, Operative Dentistry Division, Department of Restorative Dental Dr. Room D9-6, Gainesville, FL 32610, United States (e-mail: mrocha@dental.ufl.edu).
\end{abstract} \\ Sciences, University of Florida, College of Dentistry, 1395 Center
} Piracicaba Dental School, State University of Campinas, Sao Paulo, Brazil

Eur J Dent 2022;16:64-79.

\begin{abstract}
Keywords

- beam profile

- light curing units

- spectrophotometric analysis

- digital cameras

Objective This study aimed to perform the beam profile of dental light-curing units (LCUs) using mirrorless and smartphone cameras and correlate it to a camera-based laser beam profiling system.

Materials and Methods Three LCUs were evaluated (Radii Plus; Bluephase G2; and VALO Cordless). The spectral power of the LCUs was measured by using a spectrophotometer. The light emitted from the LCUs was projected onto a glass diffuser, and the images were recorded by using a mirrorless camera (NEX-F3), a smartphone (iPhone) and a camera-based beam profiler. Bandpass optical-filters were used, and for each LCU, the total spectral power output was integrated to calibrate the images. Statistical analysis was performed by digital image correlation (pixel by pixel) using Pearson's correlation ( $\alpha=0.05 ; \beta=0.2)$.

Results The beam profile images showed nonuniform radiant emittance and spectral emission distributions across all the LCUs light tip. A strong correlation was found among cameras (Pearson's $r=0.91 \pm 0.03$ with $95 \%$ confidence interval $[\mathrm{Cl}]$ : $0.88-0.94$ for the NEX-F3 and Pearson's $r=0.88 \pm 0.04$ with $95 \% \mathrm{Cl}: 0.84-0.92$ for the iPhone).

Conclusion The standard Ophir beam profile system presented the most accurate distribution, but the mirrorless and smartphone cameras presented a strong correlation in the irradiance distribution of the beam profile images. Alternative cameras can be used to perform light beam profile of dental LCUs, but caution is needed as the type of sensor, image bit depth, and image processing are important to obtain accurate results.
\end{abstract}

\section{Introduction}

Light curing units (LCUs) are used for light activation of dental adhesives, resin-based composites, resin cements, resin-modified glass ionomers, and many other light-curable

published online August 27, 2021
DOI https://doi.org/

$10.1055 / \mathrm{s}-0041-1731628$ ISSN $1305-7456$ dental materials. ${ }^{1,2}$ Frequently, manufacturers clearly state that the LCU's irradiance $\left(\mathrm{mW} / \mathrm{cm}^{2}\right)$, and for the most part of the industry, LCUs with an average irradiance higher than $1000 \mathrm{~mW} / \mathrm{cm}^{2}$ are considered decent. ${ }^{3}$ It is important to mention that the irradiance is the power $(\mathrm{mW})$ received by

(C) 2021. The Author(s).

This is an open access article published by Thieme under the terms of the Creative Commons Attribution License, permitting unrestricted use, distribution, and reproduction so long as the original work is properly cited. (https:// creativecommons. org/licenses/by/4.0/).

Thieme Medical and Scientific Publishers Pvt. Ltd. A-12, 2nd Floor, Sector 2, Noida-201301 UP, India 
a determined area $\left(\mathrm{cm}^{2}\right)$ of a resin-based material, and the radiant emittance (or radiant exitance) is the power ( $\mathrm{mW}$ ) being emitted by a determined area $\left(\mathrm{cm}^{2}\right)$ of the light tip of an LCU. ${ }^{2,3}$ Both units $\left(\mathrm{mW} / \mathrm{cm}^{2}\right)$ are the quotient of the power $(\mathrm{mW})$ of the light divided by the area $\left(\mathrm{cm}^{2}\right)$, but LCUs with the same radiant emittance $\left(\mathrm{mW} / \mathrm{cm}^{2}\right)$ might not have the same irradiance $\left(\mathrm{mW} / \mathrm{cm}^{2}\right)$.

However, even if the radiant emittance of an LCU is appropriately described, it does not fully address all significant characteristics of the LCU. Other aspects are extremally important to evaluate the quality of the light emitted, such as the LCU light tip size in comparison to the specimen size, the spectral power, and specially the light beam profile homogeneity. ${ }^{2-4}$ Thus, to systematically comprehend the emitted-light quality of an LCU, a complete characterization of the light beam emitted by the LCU is essential., 2,3,5

The light beam spectral power distribution of an LCU influences in the polymerization homogeneity of resin-based materials. ${ }^{6,7}$ For Monowave LCUs with narrowband spectral emission (within the blue wavelength spectrum only), the use of optical apparatus such as microlenses or optical fiber bundles can collimate the light creating a homogeneous light beam emission. Thus, homogeneously light activating the polymerization of resin-based materials containing only camphorquinone (CQ) as the photoinitiator system. ${ }^{8}$ For multiwave LEDs that emit more than one wavelength spectrum (within the violet and blue wavelength spectra), the physical location of the LED chips emitting the different wavelengths seems to be directly correlated with the nonuniform nature of the light beam output of these multiwave LCUs. ${ }^{9-11}$ Manufacturers' claim that the combination of multiple LEDs emitting different wavelengths (violet and blue) and different photoinitiators, such as the diphenyl(2,4,6trimethylbenzoyl)phosphine oxide (TPO) and the benzoyl germanium (Ivocerin), provide better photopolymerization for resin-based materials. ${ }^{9}$ However, one of the main obstacles is to build an LCU by using multiple LED chips with a homogeneous light beam emission. ${ }^{10,11}$

Therefore, the light beam profile is an important aspect that reveals valuable information about the LCU. A light beam profile can be defined as the power intensity plot of a light beam at a given location along with the light beam output. To characterize the light beam profile, camera-based beam profilers are often used. ${ }^{12,13}$ The camera-based beam profiler is an instrument that uses a charge-coupled device (CCD) or complementary metal-oxide-semiconductor (CMOS) camera-based detectors to measure the spatial distribution of light intensity in the cross-section of a light beam according to the conversion of the light photon flux to an electrical current-voltage signal. ${ }^{14}$ With the use of bandpass filters, the wavelength distribution of the light beam could also be detected. However, one of the main obstacles of this method is the cost of the complete instrumentation for a camera-based beam profiling system.

The high cost of a beam profiling system is related to the special CCD cameras, which includes a frame grabber card to transcript the signal, a software for controlling the frame grabber card to display beam profiles and to make respective quantitative calculations. Perhaps the most significant reason for this equipment high cost is that those commercially available beam profilers are designed to characterize industrial or medical lasers, and those lasers have an extremely high-powered light beam emission from 10 to $100 \mathrm{~W}$ in areas of 1 to $5 \mathrm{~mm}^{2}$. However, for dental LCU that uses LEDs with power emission from 1 to $5 \mathrm{~W}$ in areas of 5 to $10 \mathrm{~mm}^{2}$, it is not necessary to use equipment with that sophistication.

Thus, it would be great to have low cost equipment that dentists and researchers could get a more robust report of the beam profile of the LCUs that they have been using in their clinics or laboratories. The aim of this study was to demonstrate a method to perform the beam profile of dental LCUs by using low budget cameras and a free open-source software and correlate it to a gold-standard method using camera-based beam profiling system. The research hypothesis was that there are no differences on the beam profiling of different LCUs obtained by using mirrorless, smartphone, and standard camera-based beam profiling systems.

\section{Materials and Methods}

\section{Spectral Radiant Power}

Three LCUs were characterized in this study: Radii Plus (SDI Ltd., Bayswater, Victoria, Australia), Bluephase G2 (Ivoclar Vivadent, Schaan, Liechtenstein), and Valo Cordless (Ultradent Products Inc., South Jordan, Utah, United States). -Fig. 1 shows pictures of the light tip and a schematic representation of the chipset array of the LCUs. For each LCU, the area of light emission $\left(\mathrm{cm}^{2}\right)$ was measured by using a digital caliper by the mean of five readings of the inner diameter $(\mathrm{d})$ of the light tip and calculating the area $\left(A_{\text {circle }}\right)$ using the formula of the area of a circle $\left(A_{\text {circle }}=(d / 2) 2\right.$. The spectral radiant power $(\mathrm{mW} / \mathrm{nm})$ of the LCUs was measured five times by using a spectrophotometer with a $16-\mathrm{mm}$ diameter light collection area (MARC Light Collector, BlueLight Analytics, Halifax, Nova Scotia, Canada). ${ }^{4}$ For each LCU, the radiant emittance $\left(\mathrm{mW} / \mathrm{cm}^{2}\right)$ was calculated by the division of the radiant power $(\mathrm{mW} / \mathrm{nm})$ by the area of light emission $\left(\mathrm{cm}^{2}\right)$, and the irradiance $\left(\mathrm{mW} / \mathrm{cm}^{2}\right)$ was calculated by the division of the radiant power $(\mathrm{mW} / \mathrm{nm})$ by a predetermined circle area of $10 \mathrm{~mm}$ in diameter $\left(\mathrm{A}=0.785 \mathrm{~cm}^{2}\right)$. The irradiance values in this scenario would represent the exact amount of light delivered in hypothetical situations of light curing a molar tooth with $10 \mathrm{~mm}$ of distance mesiodistal or light curing a disk specimen with $10 \mathrm{~mm}$ in diameter for in vitro studies.

As specified by the manufacturer, ${ }^{15}$ the Radii Plus has four LED chips in the blue wavelength range with an emission peak at $450 \mathrm{~nm}$. The Bluephase G2 has four LED chips, one in the violet wavelength range with a spectral emission peak at $410 \mathrm{~nm}$ and the other three in the blue wavelength range with an emission peak at $460 \mathrm{~nm} .{ }^{16}$ The VALO Cordless light, which also has four LED chips, contains one in the violet wavelength range (spectral peak at $405 \mathrm{~nm}$ ) and the other three in the blue wavelength range, with one chip emitting at $440 \mathrm{~nm}$ and the other two at $460 \mathrm{~nm} .{ }^{17}$ The radiant emittance $\left(\mathrm{mW} / \mathrm{cm}^{2}\right)$ and the irradiance $\left(\mathrm{mW} / \mathrm{cm}^{2}\right)$ on the ultraviolet $(<380 \mathrm{~nm})$, violet $(380-420 \mathrm{~nm})$, royal-blue $(420-450 \mathrm{~nm})$, 


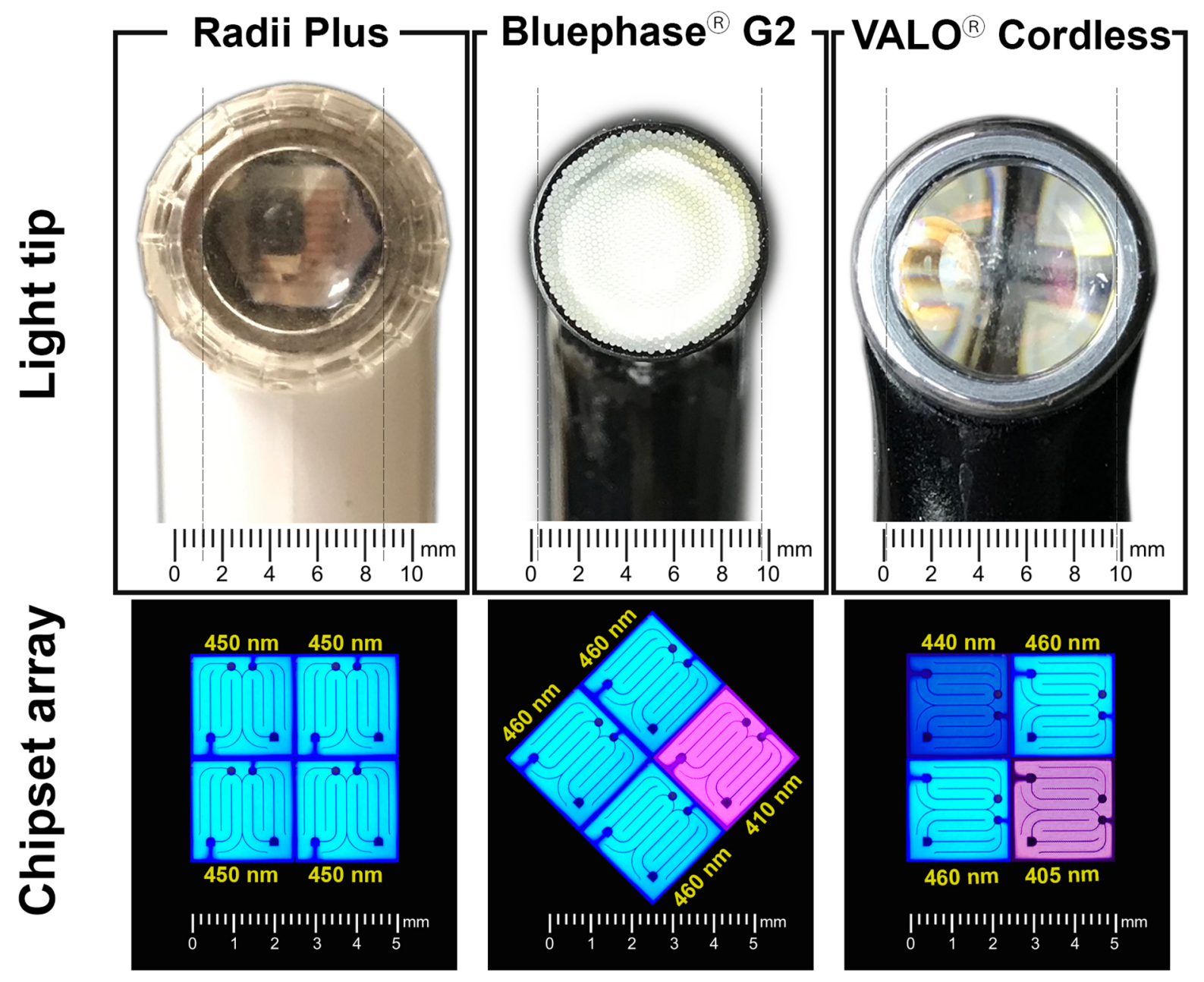

Fig. 1 Light tip illustration showing in proportional size the differences between the LCUs ([A] radii plus, [B] Bluephase G2, and [C] VALO Cordless) and LED chipset array distribution according to the number of LED chips, LED chip wavelength peak of emission, and the position of the LED chips for each LCU. The Chipset Array scheme is based on images collect from the LED chip by using a digital optical microscope (VHX-100, Keyence, Osaka, Japan). LCU, light-curing units

cyan-blue (450-495 nm), green (495-540 nm), as well as, the overall wavelength range (360-540 nm) of each LCU was calculated by integrating the radiant emittance $\left(\mathrm{mW} / \mathrm{cm}^{2}\right)$ and the irradiance $\left(\mathrm{mW} / \mathrm{cm}^{2}\right)$ versus wavelength curves obtained from the spectrophotometer.

\section{Beam Profile Image Acquisition}

A schematic representation of the set up used to make the beam profile images acquisition is showed in - Fig. 2. Each LCU was attached to an $\mathrm{x}-\mathrm{y}-\mathrm{z}$ positioning device mounted on an optical bench (450 $\times 300 \mathrm{~mm}$ breadboard, Edmund Optics, Barrington, New Jersey, United States) to standardize the positioning of the light beam in contact with a diffusive surface of a frosted diffuser glass (DG20-1500, Thorlabs, Inc.). To assess the irradiance distribution according to different wavelength range emission and to narrow the differences in the camera pixel absorption profile, bandpass filters (FB410-10, FB440-10 and FB-460-10, Thorlabs, Inc.) were placed between the diffuser glass and the cameras.
As the bandpass filters used in this study has an optical density of approximately 0.2 , no neutral density filters were necessary to attenuate the LCU light to avoid pixel intensity saturation on the images. More specific information about the bandpass filter characteristics is provided in the - Supplementary Material (available in the online version). For the Radii Plus, a bandpass filter centered first at $460 \pm$ $2 \mathrm{~nm}$ with a $10 \pm 2 \mathrm{~nm}$ full width at half maximum (FB460-10, Thorlabs, Inc.) was used to identify the LED chip with a spectrum emission peak at $460 \mathrm{~nm}$. For the Bluephase G2, a bandpass filter centered first at $410 \pm 2 \mathrm{~nm}$ with a $10 \pm 2 \mathrm{~nm}$ full width at half maximum (FB410-10, Thorlabs, Inc.) was used to identify the LED chips with a spectrum emission peak at $410 \mathrm{~nm}$. A different bandpass filter, centered at $460 \pm 2 \mathrm{~nm}$ with a $10 \pm 2 \mathrm{~nm}$ full width at half maximum (FB460-10, Thorlabs, Inc.) was used to identify the LED chips generating emission peak near $460 \mathrm{~nm}$. For the VALO Cordless, the same bandpass filters described above (FB410-10 and FB460-10) were used to identify the LED chips with spectrum emission 
peaks at 405 and $460 \mathrm{~nm}$. An additional bandpass filter, centered at $440 \pm 2 \mathrm{~nm}$ with a $10 \pm 2 \mathrm{~nm}$ full width at half maximum (FB440-10) was used to identify the LED chip having an emission peak near $440 \mathrm{~nm}$.

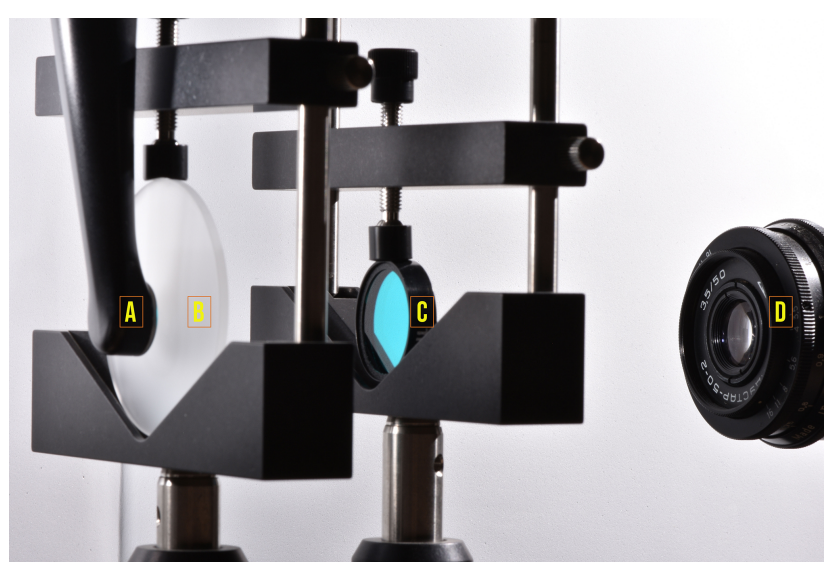

Fig. 2 Representative picture of the method setup for the collection of the beam profile data using different cameras: Each $L C U(\mathbf{A})$ was positioned perpendicular to a glass diffuser (B) target and pictures were taken by using different cameras (D) with different bandpass filter (C) between the glass diffuser and the cameras' lens; the focal distance between the camera lens and the LCU light tip was $25 \mathrm{~cm}$ for the Ophir camera, $28 \mathrm{~cm}$ for the NEX-F3 camera, and $10 \mathrm{~cm}$ for the iPhone camera. LCU, light-curing units.
The resulting images were recorded by using different cameras: a standard beam profiler CCD camera (Ophir, Model SP503U, Ophir-Spiricon, Logan, Utah, United States),, a mirrorless camera (NEX-F3, Sony Corporation, Tokyo, Japan) with $50 \mathrm{~mm}$ focal length lens and a smartphone camera (iPhone 7 Plus, Apple Inc., Cupertino, California, United States). The details of the three cameras used in this study (Ophir, NEX-F3 and iPhone) are reported in - Table $\mathbf{1}$.

For the Ophir camera, the images were captured by using the BeamGage Standard software (v.6.13.1, Ophir-Spiricon). First, the system was automatically corrected for ambient light and pixel response. Then, the LCUs were powered on and the Ophir SP503U $50 \mathrm{~mm}$ lens camera iris was adjusted to use the full dynamic range of the CCD camera without pixel intensity saturation. For the NEX-F3, a sequence of images was captured manually with ISO 200 an aperture of $\mathrm{f} / 32$ and shutters speed from $1 / 4,000$ to $1 / 200$. For the iPhone, a series of images was captured manually by using the application software Adobe Photoshop Lightroom CC (v. 3.4.0 F59BE2, Adobe, San Jose, California, United States) to adjust the iPhone 7 Plus rear camera to an ISO 25, an aperture of $\mathrm{f} / 1.8$, and a shutter speed from $1 / 8,000$ to $1 / 200$.

The NEX-F3 and the iPhone cameras images were captured in the RAW format with minimally processed data from the image sensor to achieve the maximum dynamic range of both cameras. The images with different times of exposure

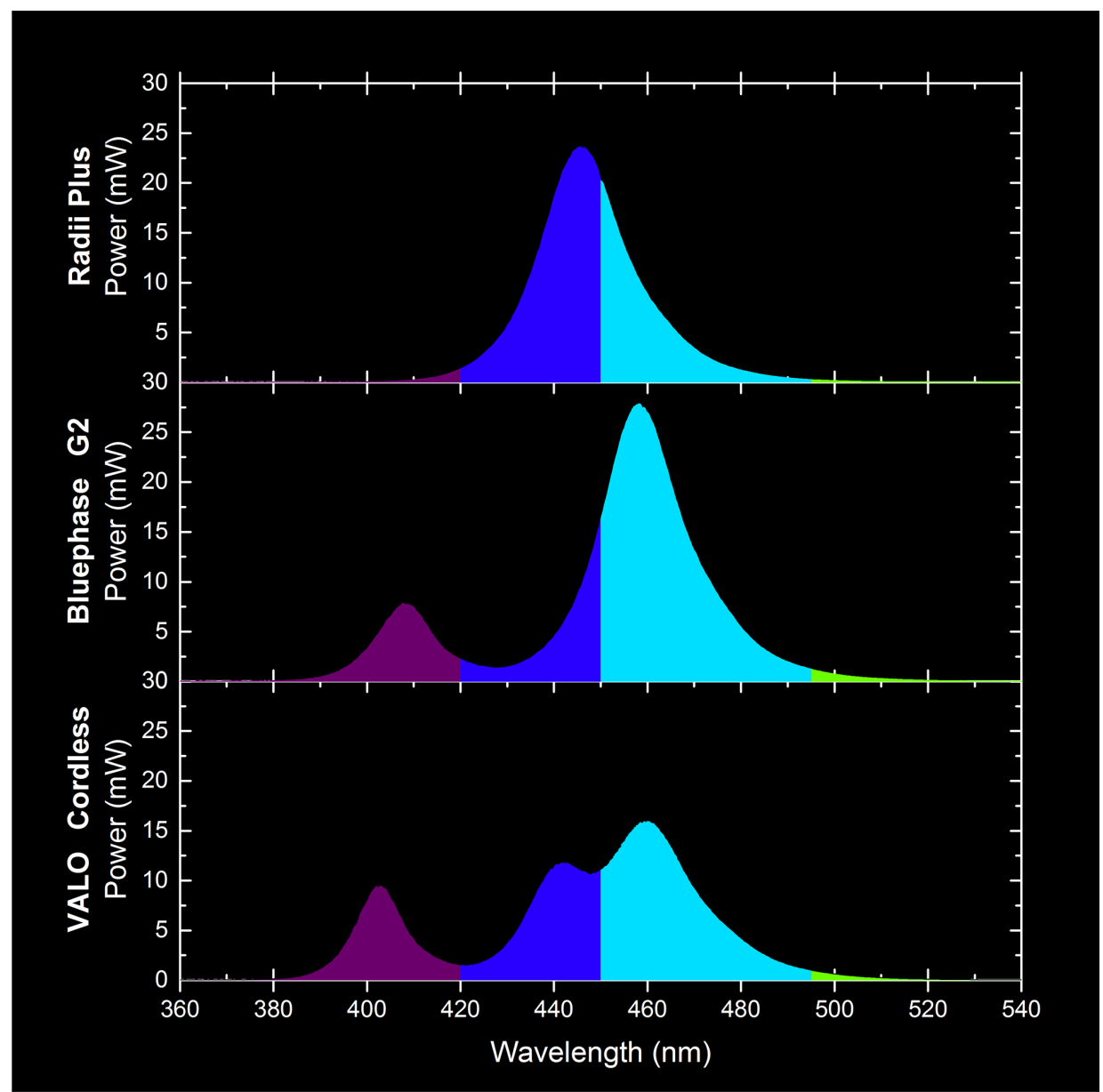

Fig. 3 Spectral radiant power (mW/nm) of the Radii Plus, Bluephase G2, and VALO Cordless. 


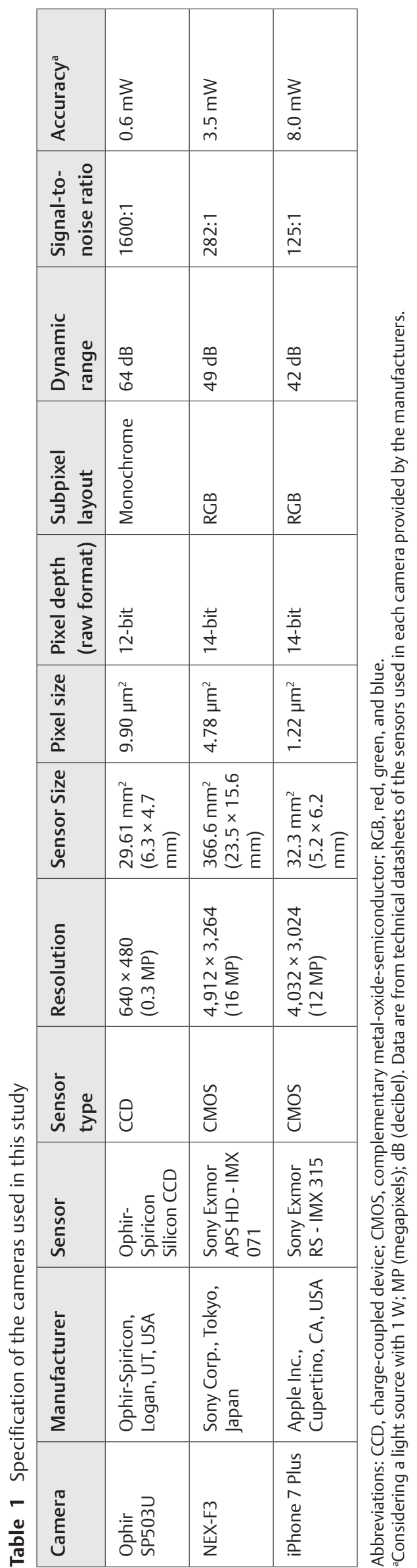

accordingly to the different shutter speed settings for each camera were examined by histogram analysis of the pixel intensity using the Fiji software to ensure that the images obtained were not saturated (pixel intensity over 65,536 since the images were taken in 16 bit). The images in which the pixel intensity reached the values closer 65,536 and lower than to 65,535 were chosen to be used as the beam profile images.

Each image was individually calibrated according to the power $(\mathrm{mW})$ recorded with the MARC LC for each LCU. The spectral irradiance for violet (peak at $405 \mathrm{~nm}$ ), royal-blue (peak at $440 \mathrm{~nm}$ ), and cyan-blue (peak at $460 \mathrm{~nm}$ ) wavelength regions were recorded and calibrated on separate images with the aid of appropriate bandpass filters. For the Ophir camera, the average power of each LCU was entered into the Beamgage software, and the irradiance distribution was determined by the average pixel intensity distribution interpolated with the size of the area of the light beam. As the Beamgage software offers limited options to customize the graphs and to manipulate the data, the 307,200 calibrated data points from the $640 \times 480$ matrix array were exported into a scientific graphing and data analysis software (Origin Pro, OriginLab Co., Northampton, Massachusetts, United States).

For the NEX-F3, the images files in the Sony Alpha Raw format (ARW) were transferred to a personal computer, and the images files in ARW were converted into a 16-bit Tagged Image File Format (TIF). The converted images were imported into a free open-source image processing and data analysis software (Fiji, ImageJ, National Institute of Health, Bethesda, Maryland, United States). ${ }^{18}$ The same process was performed for the iPhone images. The iPhone images in the Adobe Digital Negative Raw Image file format (DNG) were also converted to a 16-bit TIF file. For NEX-F3 and iPhone cameras, the average power of each LCU was entered into the Fiji software, and the irradiance distribution was determined by the average pixel intensity distribution interpolated with the size of the area of the light beam. Similar to the Beamgage software, the Fiji software offers limited options to customize graphs and manipulate the data. Thus, the calibrated data points were exported into a scientific graphing and data analysis software (Origin Pro, OriginLab Co.).

\section{Statistical Analysis}

Statistical analysis was performed by digital image correlation. Each calibrated beam profile image was transformed into an XYZ matrix array, where the X- and Y-axis represent the location of the pixel and $Z$ represents the calibrated irradiance value (according to the 16-bit grayscale pixel intensity). The data were entered into statistical analysis software (Stata/MP 13, StataCorp, College Station, Texas, United States). For each LCU beam profile image with different wavelength spectrum bandpass filter, the correlation was performed between the control group camera (Ophir) and the mirrorless camera (NEX-F3) and the smartphone camera (iPhone). A correlation analysis was performed by using Pearson's correlation with $\alpha=0.05$ and $\beta=0.2$. 


\section{Results}

\section{Spectral Radiant Power}

- Table 2 shows the total power output ( $\mathrm{mW})$, the area of light emission $\left(\mathrm{cm}^{2}\right)$, radiant emittance $\left(\mathrm{mW} / \mathrm{cm}^{2}\right)$, and the irradiance $\left(\mathrm{mW} / \mathrm{cm}^{2}\right)$ over a $0.765 \mathrm{~cm}^{2}$ area according to the different wavelength ranges. - Fig. 3 illustrates the spectral power ( $\mathrm{mW}$ ) distribution according to each wavelength ( $\mathrm{nm}$ ). The Radii Plus had total power of $607 \pm 9.3 \mathrm{~mW}$ within area of emission of $0.442 \mathrm{~cm}^{2}$ resulting an average radiant emittance of $1,378 \pm 8.2 \mathrm{~mW} / \mathrm{cm}^{2}$ and an average irradiance of $776 \pm 4.6 \mathrm{~mW} / \mathrm{cm}^{2}$. As expected, the majority of the light emission of this monowave LCU was inside "royal-blue" and "cyan-blue" wavelength range with a single peak at $454 \mathrm{~nm}$. For the Bluephase G2 in the high-power mode, the total power was $843 \pm 7.8 \mathrm{~mW}$ with an area of emission of $0.635 \mathrm{~cm}^{2}$ resulting in an average radiant emittance of $1,305 \pm 9.4 \mathrm{~mW} / \mathrm{cm}^{2}$ and an average irradiance of 1,056 \pm $7.6 \mathrm{~mW} / \mathrm{cm}^{2}$. As the Bluephase $\mathrm{G} 2$ is a dual peak multiwave LCU, with one violet LED chip, and three blue LED chips, the light emission of this LCU was inside the "violet," "royal-blue," and "cyan-blue" wavelength ranges with peaks at $408 \mathrm{~nm}$ (violet) and $458 \mathrm{~nm}$ (cyan-blue). For the VALO Cordless in the standard mode, the total power was $843 \pm 7.8 \mathrm{~mW}$ with an area of emission of $0.635 \mathrm{~cm}^{2}$ resulting in an average radiant emittance of $1,066 \pm 2.4 \mathrm{~mW} / \mathrm{cm}^{2}$ and an average irradiance of $938 \pm 2.1 \mathrm{~mW} / \mathrm{cm}^{2}$. As the VALO Cordless is a triple peak multiwave LCU, with one violet LED chip, one royal-blue LED chip and two blue LED chips, the light emission of this LCU was inside the "violet," "royal-blue," and "cyan-blue" wavelength ranges with peaks at $403 \mathrm{~nm}$ (violet), $442 \mathrm{~nm}$ (royal-blue), and $458 \mathrm{~nm}$ (cyan-blue).

\section{Light-Curing Units Beam Profile}

-Figs. 4 to 8 show the 2D and 3D beam profile images for the different LCUs using the Ophir, NEX-F3, and iPhone cameras. All the images were calibrated accordingly to the radiant emittance $\left(\mathrm{mW} / \mathrm{cm}^{2}\right)$ for each respective LCU. Also, the active area of emission $\left(\mathrm{cm}^{2}\right)$, the radiant emittance $\left(\mathrm{mW} / \mathrm{cm}^{2}\right)$, and irradiance $\left(\mathrm{mW} / \mathrm{cm}^{2}\right)$ distribution of each LCU according to the different wavelength ranges are described in - Table 3.

-Fig. 4 shows the 2D and 3D beam profile images for the Radii Plus using the Ophir, NEX-F3, and iPhone cameras. The Radii Plus had a higher light emission at the center part of the light tip and a lower radiant emittance on the boundaries of the light tip emission. Using the Ophir camera for the Radii Plus beam profile analysis, it was detected an average radiant emittance of $1,355 \mathrm{~mW} / \mathrm{cm}^{2}$, with a maximum

Table 2 Mean \pm standard deviation of the total power output ( $\mathrm{mW}$ ), active area of light emission (cm2), radiant emittance (mW/ $\mathrm{cm} 2)$, and irradiance $(\mathrm{mW} / \mathrm{cm} 2)$ according to the different spectral ranges

\begin{tabular}{|c|c|c|c|c|}
\hline \multirow{2}{*}{\multicolumn{2}{|c|}{ Light curing unit }} & \multirow[t]{2}{*}{ Radii plus } & Bluephase G2 & VALO cordless \\
\hline & & & $\begin{array}{l}\text { (High power } \\
\text { mode) }\end{array}$ & (Standard mode) \\
\hline \multicolumn{2}{|l|}{ Area of light emission $\left(\mathrm{cm}^{2}\right)$} & $0.442 \pm 0.002$ & $0.635 \pm 0.001$ & $0.691 \pm 0.001$ \\
\hline \multirow[t]{3}{*}{ Total $(360-540 \mathrm{~nm})$} & Power (mW) & $607 \pm 9.3$ & $843 \pm 7.8$ & $737 \pm 9.1$ \\
\hline & Radiant Emittance $\left(\mathrm{mW} / \mathrm{cm}^{2}\right)$ & $1,378 \pm 8.2$ & $1,305 \pm 9.4$ & $1,066 \pm 2.4$ \\
\hline & Irradiance $\left(\mathrm{mW} / \mathrm{cm}^{2}\right)$ & $776 \pm 4.6$ & $1,056 \pm 7.6$ & $938 \pm 2.1$ \\
\hline \multirow[t]{3}{*}{ Ultra-violet (<380 nm) } & Power (mW) & $0.6 \pm 0.1$ & $1 \pm 0.5$ & $1 \pm 0.6$ \\
\hline & Radiant Emittance $\left(\mathrm{mW} / \mathrm{cm}^{2}\right)$ & $1 \pm 0.1$ & $2 \pm 0.3$ & $2 \pm 0.2$ \\
\hline & Irradiance $\left(\mathrm{mW} / \mathrm{cm}^{2}\right)$ & $<1$ & $2 \pm 0.2$ & $2 \pm 0.2$ \\
\hline \multirow[t]{3}{*}{ Violet (380-420 nm) } & Power (mW) & $9 \pm 0.2$ & $123 \pm 3.9$ & $144 \pm 7.1$ \\
\hline & Radiant Emittance $\left(\mathrm{mW} / \mathrm{cm}^{2}\right)$ & $81 \pm 1.4$ & $190 \pm 4.1$ & $210 \pm 3.1$ \\
\hline & Irradiance $\left(\mathrm{mW} / \mathrm{cm}^{2}\right)$ & $46 \pm 0.8$ & $154 \pm 3.3$ & $185 \pm 2.7$ \\
\hline \multirow[t]{3}{*}{ Royal-blue (420-450 nm) } & Power (mW) & $365 \pm 7.6$ & $134 \pm 5.2$ & $211 \pm 3.3$ \\
\hline & Radiant Emittance $\left(\mathrm{mW} / \mathrm{cm}^{2}\right)$ & $1,072 \pm 4.0$ & $207 \pm 3.2$ & $305 \pm 3.1$ \\
\hline & Irradiance $\left(\mathrm{mW} / \mathrm{cm}^{2}\right)$ & $604 \pm 2.2$ & $167 \pm 2.6$ & $268 \pm 2.7$ \\
\hline \multirow[t]{3}{*}{ Cyan-Blue (450-495 nm) } & Power (mW) & $230 \pm 9.8$ & $572 \pm 3.1$ & $369 \pm 5.2$ \\
\hline & Radiant Emittance $\left(\mathrm{mW} / \mathrm{cm}^{2}\right)$ & $219 \pm 4.6$ & $885 \pm 4.5$ & $534 \pm 6.7$ \\
\hline & Irradiance $\left(\mathrm{mW} / \mathrm{cm}^{2}\right)$ & $123 \pm 2.6$ & $716 \pm 3.6$ & $470 \pm 5.9$ \\
\hline \multirow{3}{*}{$\begin{array}{l}\text { Blue (420-495 nm) (Royal + } \\
\text { Cyan) }\end{array}$} & Power (mW) & $595 \pm 6.4$ & $706 \pm 2.6$ & $580 \pm 4.2$ \\
\hline & Radiant Emittance $\left(\mathrm{mW} / \mathrm{cm}^{2}\right)$ & $1,346 \pm 4.5$ & $1,112 \pm 8.1$ & $839 \pm 3.6$ \\
\hline & Irradiance $\left(\mathrm{mW} / \mathrm{cm}^{2}\right)$ & $778 \pm 8.3$ & $923 \pm 8.7$ & $758 \pm 3.4$ \\
\hline \multirow[t]{3}{*}{ Green (495-540 nm) } & Power (mW) & $3 \pm 0.4$ & $13 \pm 1.8$ & $11 \pm 2.6$ \\
\hline & Radiant Emittance $\left(\mathrm{mW} / \mathrm{cm}^{2}\right)$ & $4 \pm 0.5$ & $20 \pm 1.2$ & $16 \pm 0.9$ \\
\hline & Irradiance $\left(\mathrm{mW} / \mathrm{cm}^{2}\right)$ & $2 \pm 0.3$ & $16 \pm 1.0$ & $14 \pm 0.8$ \\
\hline
\end{tabular}




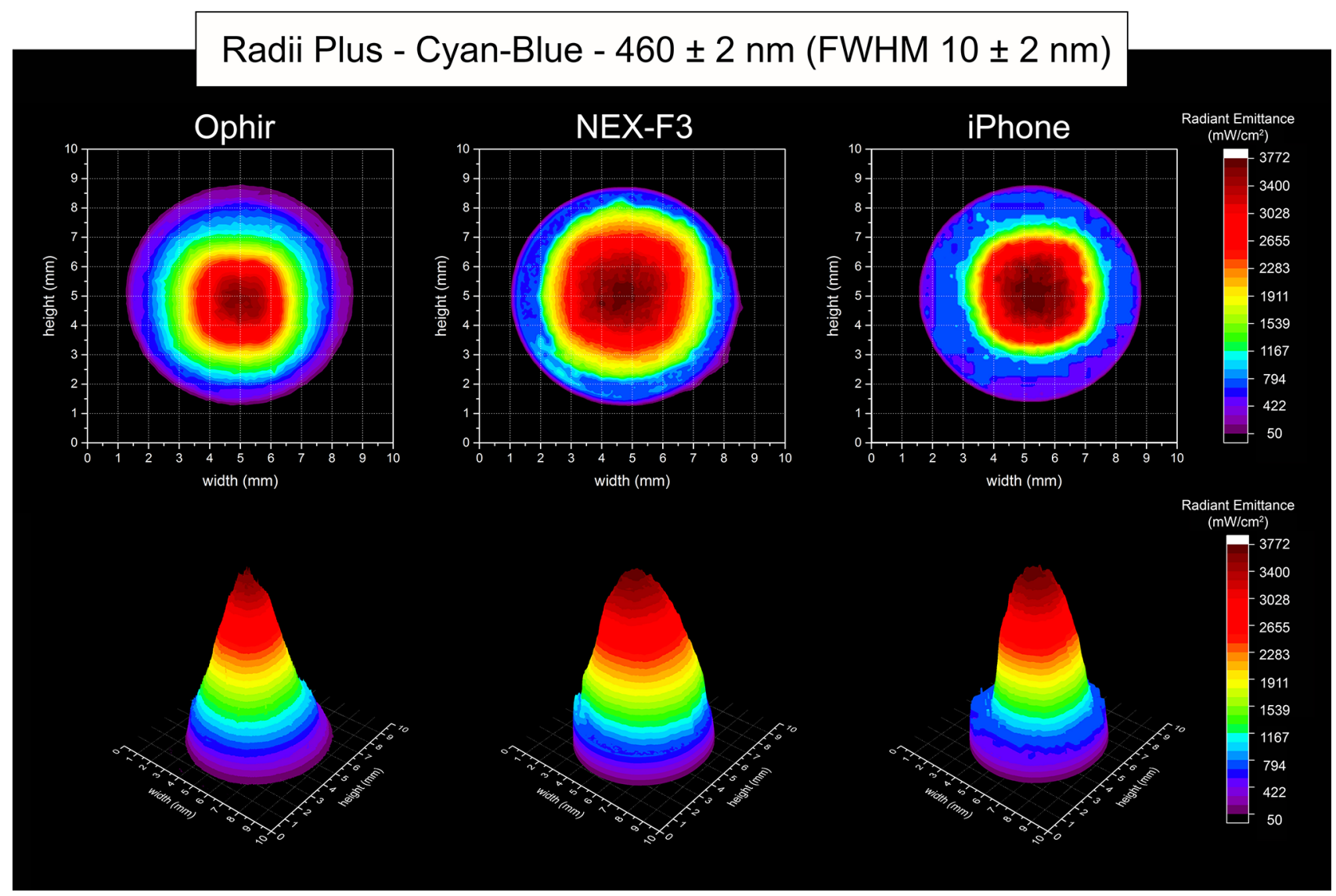

Fig. 4 Using the Ophir, NEX-F3 and iPhone cameras: 2D and 3D beam profile images of the Radii plus with the $460 \pm 2 \mathrm{~nm}$, full-width at half-maximum $10 \pm 2 \mathrm{~nm}$ bandpass filter showing the radiant emittance distribution.

radiant emittance of $3,745 \mathrm{~mW} / \mathrm{cm}^{2}$ and a minimum radiant emittance of $154 \mathrm{~mW} / \mathrm{cm}^{2}$, inside an active area of emission of $0.423 \mathrm{~cm}^{2}$ (- Table 3). Using the NEX-F3 camera for the Radii Plus beam profile analysis, it was detected an average radiant emittance of $1,389 \mathrm{~mW} / \mathrm{cm}^{2}$, with a maximum radiant emittance of $3,466 \mathrm{~mW} / \mathrm{cm}^{2}$ and a minimum radiant emittance of $186 \mathrm{~mW} / \mathrm{cm}^{2}$, inside an active area of emission of $0.425 \mathrm{~cm}^{2}$. Using the iPhone camera for the Radii Plus beam profile analysis, it was detected an average radiant emittance of $1,298 \mathrm{~mW} / \mathrm{cm}^{2}$, with a maximum radiant emittance of $3,116 \mathrm{~mW} / \mathrm{cm}^{2}$ and a minimum radiant emittance of $134 \mathrm{~mW} / \mathrm{cm}^{2}$, inside an active area of emission of $0.411 \mathrm{~cm}^{2}$.

-Figs. 5 and 6 show the 2D and 3D beam profile images for the Bluephase G2 using the Ophir, NEX-F3, and iPhone cameras within the violet spectrum and the blue spectrum, respectively. For the Bluephase G2, an area with higher light emission was detected on the boundaries of the light tip. However, according to the LED chips distribution, the violet and blue spectrum were placed in different regions across the light tip. Within the violet spectrum $(410 \pm 2 \mathrm{~nm}$, FWHM $10 \pm 2 \mathrm{~nm}$ ), an area of higher radiant emittance was detected on the left side of the light tip, where the violet LED chip is located inside the body of the LCU next to an optical reflector. Using the Ophir camera for the analysis of the Bluephase G2 inside the violet spectrum (410 \pm
$2 \mathrm{~nm}$, FWHM $10 \pm 2 \mathrm{~nm}$ ), an average radiant emittance of $195 \mathrm{~mW} / \mathrm{cm}^{2}$ was detected, with a maximum radiant emittance of $584 \mathrm{~mW} / \mathrm{cm}^{2}$ and a minimum radiant emittance of $50 \mathrm{~mW} / \mathrm{cm}^{2}$, inside an active area of emission of $0.547 \mathrm{~cm}^{2}$ (-Table 3). When the NEX-F3 camera was used for the beam profile analysis of the Bluephase G2 inside the violet spec$\operatorname{trum}(410 \pm 2 \mathrm{~nm}, \mathrm{FWHM} 10 \pm 2 \mathrm{~nm})$, an average radiant emittance of $200 \mathrm{~mW} / \mathrm{cm}^{2}$ was detected, with a maximum radiant emittance of $492 \mathrm{~mW} / \mathrm{cm}^{2}$ and a minimum radiant emittance of $50 \mathrm{~mW} / \mathrm{cm}^{2}$, inside an active area of emission of $0.545 \mathrm{~cm}^{2}$. For the iPhone camera, the Bluephase G2 inside the violet spectrum ( $410 \pm 2 \mathrm{~nm}$, FWHM $10 \pm 2 \mathrm{~nm}$ ) beam profile analysis detected an average radiant emittance of $194 \mathrm{~mW} / \mathrm{cm}^{2}$, with a maximum radiant emittance of $382 \mathrm{~mW} / \mathrm{cm}^{2}$ and a minimum radiant emittance of $50 \mathrm{~mW} / \mathrm{cm}^{2}$, inside an active area of emission of $0.608 \mathrm{~cm}^{2}$.

Considering the beam profile analysis of the Bluephase G2 within the blue spectrum $(460 \pm 2 \mathrm{~nm}$, FWHM $10 \pm$ $2 \mathrm{~nm}$ ), an area of higher radiant emittance on the top side of the light tip was detected, where one of the LED chips emitting blue light is located inside the body of the LCU next to an optical reflector. The Ophir camera for the Bluephase G2 inside the blue spectrum ( $460 \pm 2$ nm, FWHM $10 \pm 2 \mathrm{~nm}$ ) beam profile analysis detected an average radiant emittance of $1,092 \mathrm{~mW} / \mathrm{cm}^{2}$, with a maximum radiant emittance of $2,977 \mathrm{~mW} / \mathrm{cm}^{2}$ and a minimum radiant 


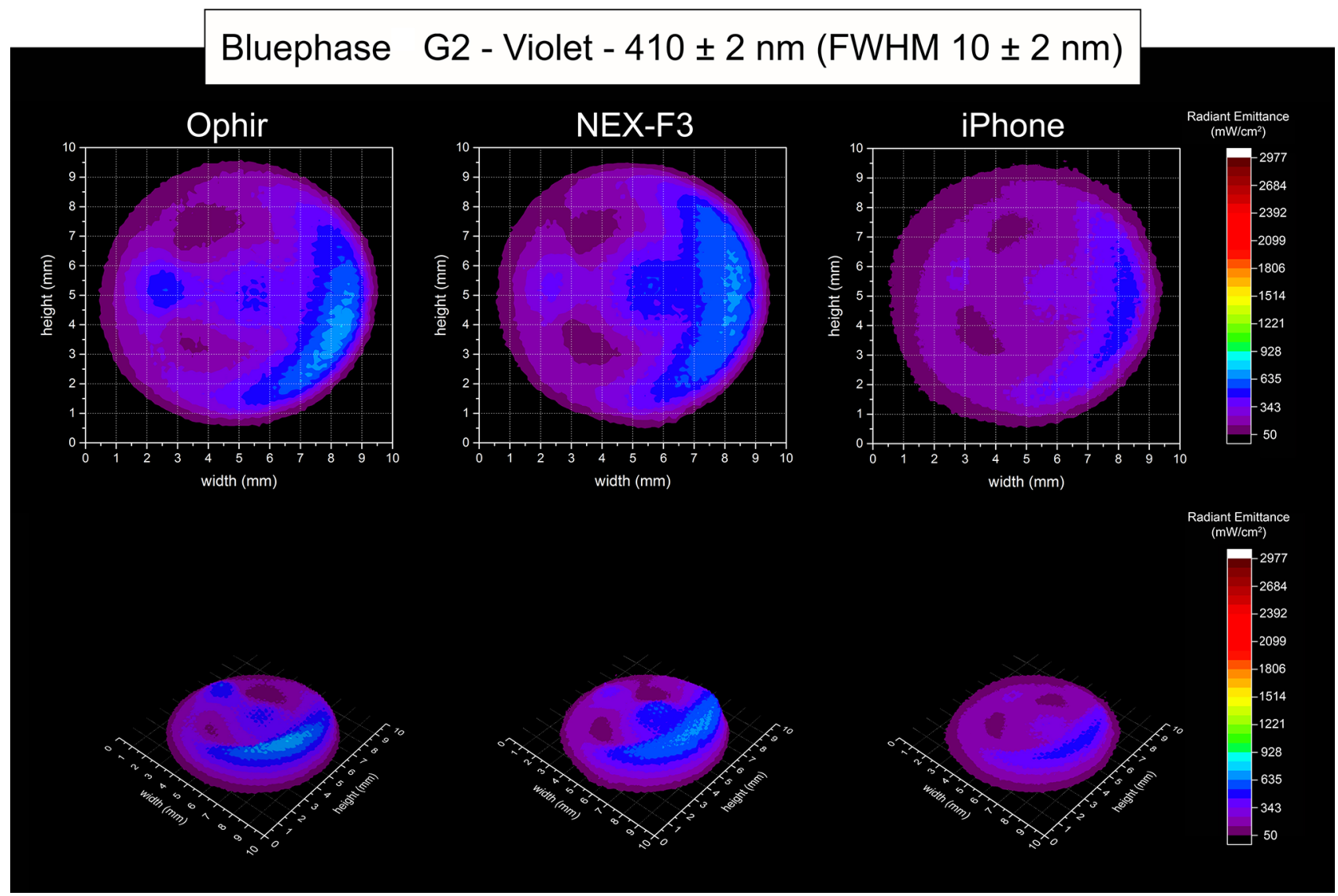

Fig. 5 Using the Ophir, NEX-F3 and iPhone cameras: 2D and 3D beam profile images of the Bluephase G2 with the $410 \pm 2 \mathrm{~nm}$, full-width at half-maximum $10 \pm 2 \mathrm{~nm}$ bandpass filter showing the radiant emittance distribution.

emittance of $130 \mathrm{~mW} / \mathrm{cm}^{2}$, inside an active area of emission of $0.589 \mathrm{~cm}^{2}$ (-Table 3). The NEX-F3 camera for the Bluephase G2 inside the blue spectrum ( $460 \pm 2 \mathrm{~nm}$, FWHM $10 \pm 2 \mathrm{~nm}$ ) beam profile analysis detected an average radiant emittance of $1089 \mathrm{~mW} / \mathrm{cm}^{2}$, with a maximum radiant emittance of $2,253 \mathrm{~mW} / \mathrm{cm}^{2}$ and a minimum radiant emittance of $109 \mathrm{~mW} / \mathrm{cm}^{2}$, inside an active area of emission of $0.604 \mathrm{~cm}^{2}$. The iPhone camera for the Bluephase G2 inside the blue spectrum ( $460 \pm 2 \mathrm{~nm}$, FWHM $10 \pm 2 \mathrm{~nm}$ ) beam profile analysis detected an average radiant emittance of $1,094 \mathrm{~mW} / \mathrm{cm}^{2}$, with a maximum radiant emittance of $2,350 \mathrm{~mW} / \mathrm{cm}^{2}$ and a minimum radiant emittance of $112 \mathrm{~mW} / \mathrm{cm}^{2}$, inside an active area of emission of $0.603 \mathrm{~cm}^{2}$.

-Figs. 7 to 9 show the 2D and 3D beam profile images for the VALO Cordless using the Ophir, NEX-F3, and iPhone cameras within the violet spectrum, the royal-blue spectrum and cyan-blue spectrum), respectively. The VALO Cordless had a higher light emission on the boundaries of the light tip emission. However, according to the distribution of the LED chips, the violet, the royal-blue, and the cyan-royal blue spectra were located in different regions of the light tip.

Within the violet spectrum $(410 \pm 2 \mathrm{~nm}$, FWHM $10 \pm$ $2 \mathrm{~nm}$ ), the VALO Cordless had an area of higher radiant emittance on the right-bottom side of the light tip, where the violet LED chip is located inside the head of the LCU. The Ophir camera for the VALO Cordless inside the violet spectrum ( $410 \pm 2 \mathrm{~nm}$, FWHM $10 \pm 2 \mathrm{~nm}$ ) beam profile analysis detected an average radiant emittance of $209 \mathrm{~mW} / \mathrm{cm}^{2}$, with a maximum radiant emittance of $555 \mathrm{~mW} / \mathrm{cm}^{2}$ and a minimum radiant emittance of $50 \mathrm{~mW} / \mathrm{cm}^{2}$, inside an active area of emission of $0.468 \mathrm{~cm}^{2}$ (-Table 3). The NEX-F3 camera for the VALO Cordless inside the violet spectrum $(410 \pm 2 \mathrm{~nm}$, FWHM $10 \pm 2 \mathrm{~nm}$ ) beam profile analysis detected an average radiant emittance of $210 \mathrm{~mW} / \mathrm{cm}^{2}$, with a maximum radiant emittance of $453 \mathrm{~mW} / \mathrm{cm}^{2}$ and a minimum radiant emittance of $50 \mathrm{~mW} / \mathrm{cm}^{2}$, inside an active area of emission of $0.433 \mathrm{~cm}^{2}$. The iPhone camera for the VALO Cordless inside the violet spectrum $(410 \pm 2 \mathrm{~nm}$, FWHM $10 \pm 2 \mathrm{~nm})$ beam profile analysis detected an average radiant emittance of $210 \mathrm{~mW} / \mathrm{cm}^{2}$, with a maximum radiant emittance of $437 \mathrm{~mW} / \mathrm{cm}^{2}$ and a minimum radiant emittance of $50 \mathrm{~mW} / \mathrm{cm}^{2}$, inside an active area of emission of $0.465 \mathrm{~cm}^{2}$.

Inside the royal-blue spectrum $(440 \pm 2 \mathrm{~nm}$, FWHM $10 \pm$ $2 \mathrm{~nm}$ ), the VALO Cordless had an area of higher radiant emittance on the top and right side of the light tip, where one of the royal-blue LED chip is located inside the body of the LCU. 


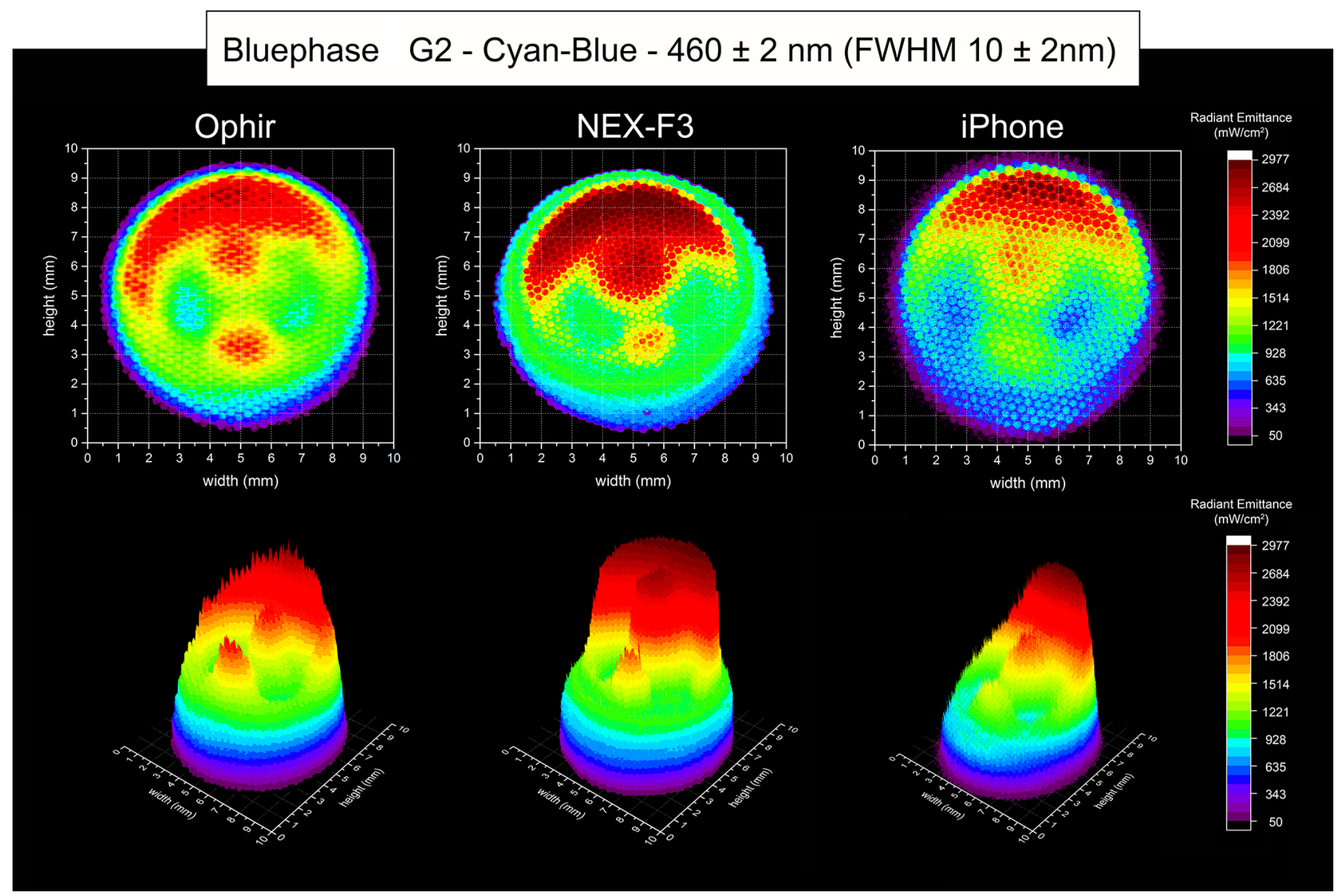

Fig. 6 Using the Ophir, NEX-F3 and iPhone cameras: 2D and 3D beam profile images of the Bluephase G2 with the $460 \pm 2 \mathrm{~nm}$, full-width at half-maximum $10 \pm 2 \mathrm{~nm}$ bandpass filter showing the radiant emittance distribution.

The Ophir camera for the VALO Cordless inside the blue spectrum (440 $\pm 2 \mathrm{~nm}$, FWHM $10 \pm 2 \mathrm{~nm}$ ) beam profile analysis detected an average radiant emittance of $515 \mathrm{~mW} / \mathrm{cm}^{2}$, with a maximum radiant emittance of $1,075 \mathrm{~mW} / \mathrm{cm}^{2}$ and a minimum radiant emittance of $106 \mathrm{~mW} / \mathrm{cm}^{2}$ inside an active area of emission of $0.523 \mathrm{~cm}^{2}$. The NEX-F3 camera for the VALO Cordless inside the royal-blue spectrum $(440 \pm 2 \mathrm{~nm}$, FWHM $10 \pm 2 \mathrm{~nm}$ ) beam profile analysis detected an average radiant emittance of $514 \mathrm{~mW} / \mathrm{cm}^{2}$, with a maximum radiant emittance of $819 \mathrm{~mW} / \mathrm{cm}^{2}$ and a minimum radiant emittance of $133 \mathrm{~mW} / \mathrm{cm}^{2}$, inside an active area of emission of $0.608 \mathrm{~cm}^{2}$. The iPhone camera for the VALO Cordless inside the royal-blue spectrum ( $440 \pm 2 \mathrm{~nm}$, FWHM $10 \pm 2 \mathrm{~nm}$ ) beam profile analysis detected an average radiant emittance of $514 \mathrm{~mW} / \mathrm{cm}^{2}$, with a maximum radiant emittance of $887 \mathrm{~mW} / \mathrm{cm}^{2}$ and a minimum radiant emittance of $135 \mathrm{~mW} / \mathrm{cm}^{2}$, inside an active area of emission of $0.574 \mathrm{~cm}^{2}$.

Within the cyan-blue spectrum, the VALO Cordless had an area of higher radiant emittance on the top-right and bottom left side of the light tip, where each of two blue LED chips are located inside the head of the LCU. The Ophir camera for the VALO Cordless inside the cyan-blue spectrum (460 $\pm 2 \mathrm{~nm}$, FWHM $10 \pm 2 \mathrm{~nm}$ ) beam profile analysis detected an average radiant emittance of $838 \mathrm{~mW} / \mathrm{cm}^{2}$, with a maximum radiant emittance of $2,106 \mathrm{~mW} / \mathrm{cm}^{2}$ and a minimum irradiance of $117 \mathrm{~mW} / \mathrm{cm}^{2}$, inside an active area of emission of $0.609 \mathrm{~cm}^{2}$ (-Table 3). The NEX-F3 camera for the VALO Cordless inside the cyan-blue spectrum ( $460 \pm 2 \mathrm{~nm}$, FWHM $10 \pm 2 \mathrm{~nm}$ ) beam profile analysis detected an average radiant emittance of $839 \mathrm{~mW} / \mathrm{cm}^{2}$, with a maximum radiant emittance of $2,115 \mathrm{~mW} / \mathrm{cm}^{2}$ and a minimum radiant emittance of $120 \mathrm{~mW} / \mathrm{cm}^{2}$, inside an active area of emission of $0.614 \mathrm{~cm}^{2}$. The iPhone camera for the VALO Cordless inside the cyan-blue spectrum ( $460 \pm 2 \mathrm{~nm}$, FWHM $10 \pm 2 \mathrm{~nm}$ ) beam profile analysis detected an average radiant emittance of $839 \mathrm{~mW} / \mathrm{cm}^{2}$, with a maximum irradiance of $2,181 \mathrm{~mW} / \mathrm{cm}^{2}$ and a minimum radiant emittance of $50 \mathrm{~mW} / \mathrm{cm}^{2}$, inside an active area of emission of $0.613 \mathrm{~cm}^{2}$.

- Table 4 shows the Pearson's correlation of the images pixel intensities according to the $\mathrm{x}$ and $\mathrm{y}$ position using the NEX-F3 and iPhone cameras in comparison to the Ophir camera. A strong correlation in the pixel intensity distribution was found between the Ophir camera and the NEX-F3 camera (Pearson's $r=0.91 \pm 0.03$ with 95\% CI: 0.88-0.94) and as well between the Ophir camera and the iPhone camera (Pearson's $r=0.88 \pm 0.04$ with $95 \%$ CI: $0.84-0.92$ for the iPhone).

\section{Discussion}

The research hypothesis that the mirrorless and smartphone cameras would have the same performance as the camera-based beam profiling systems was accepted. 


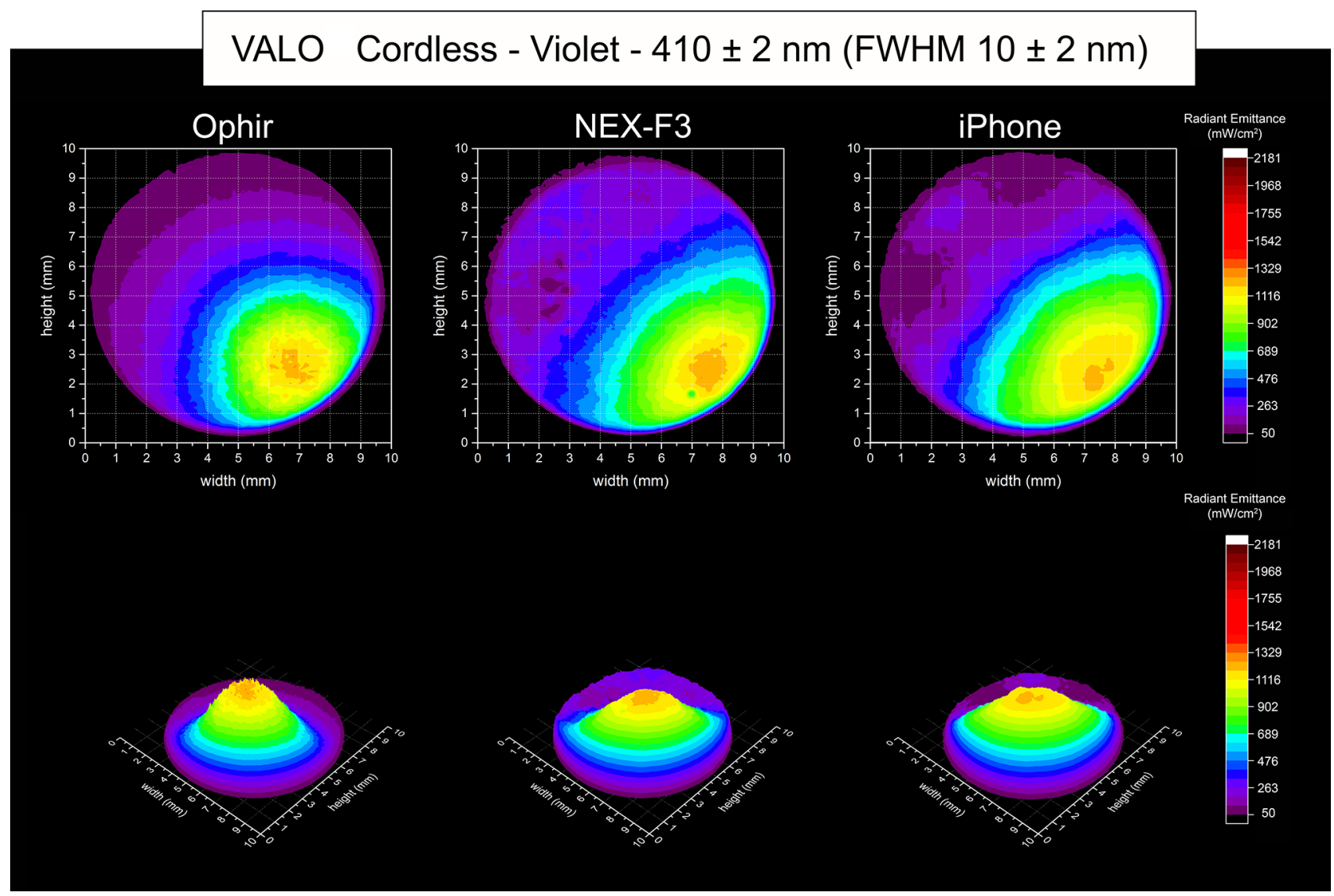

Fig. 7 Using the Ophir, NEX-F3 and iPhone cameras: 2D and 3D beam profile images of the VALO Cordless with the $410 \pm 2 \mathrm{~nm}$, full-width at half-maximum $10 \pm 2 \mathrm{~nm}$ bandpass filter showing the radiant emittance distribution.

As mentioned in this study introduction, the Ophir camera-based laser beam profiling system and other elaborated beam profile systems are considered the best quality scientific equipment regarding the characterization of light beams of medical and industrial laser. Those beam profiling systems are proven to be also reliable to characterize the light beam profile of dental LCUs..$^{1,2,7}$ However, in terms of characterizing a dental LCU, the use of more accessible technologies can provide results with similar quality but at low cost. Nevertheless, it is important to state that there are some differences between the Ophir camera and the NEX-F3 and the iPhone cameras used in this study. Indeed, these differences found in the NEX-F3 camera (mirrorless) and in the iPhone (Smartphone) camera might be the same differences observed in other models of mirrorless and smartphone cameras, since these electronical devices share similar camera technology. ${ }^{19}$

The first difference is that the Ophir camera uses a charge couple devices (CCD) sensor while the NEX-F3 and the iPhone cameras use a complementary metal-oxide semiconductor (CMOS) sensor. The CCD and CMOS sensors operate quite similar using an array of photodetectors. Then, when a photon from the LCU reaches the photodetector, a photoelectric conversion occurs generating electrons in each photodetector. ${ }^{20-22}$ The number of electrons generated in each photodetector is proportional to the number of photons that have reached the sensor. However, the difference between CCD and CMOS sensors is in the conversion of the analog electron signal in each photodetector to a digital signal in a form of image pixel..$^{20}$ Basically, for the CMOS sensor, each photodetector has its motherboard processor located at each pixel, which converts the analog to the digital signal. On the other hand, the motherboard processor of a CCD sensor that converts the analog to the digital signal is located nearest to the edge of the sensor. ${ }^{21}$ Thus, the photodetectors on the edge of the sensor transfer their electrons to the motherboard processor. However, all other photodetectors transfer their electron charge to the neighboring photodetector that is closer to the motherboard and the process continues when these photodetectors transfer the electrons to the motherboard processor. ${ }^{20-22}$ Once the motherboard processes the analog signal and transforms it into a digital signal, this process continues until all electrons captured in an image are detected and converted into digital. In this way, the electrons are removed from the sensor one photodetector at a time. ${ }^{20}$ The fact is that for many years, CCD sensor was the predominant sensor used in laser beam profiling applications. Also, one of the foremost reasons behind the CCD use was their lower noise level, which means that the images are smoother and the pixel intensity values are more accurate. 


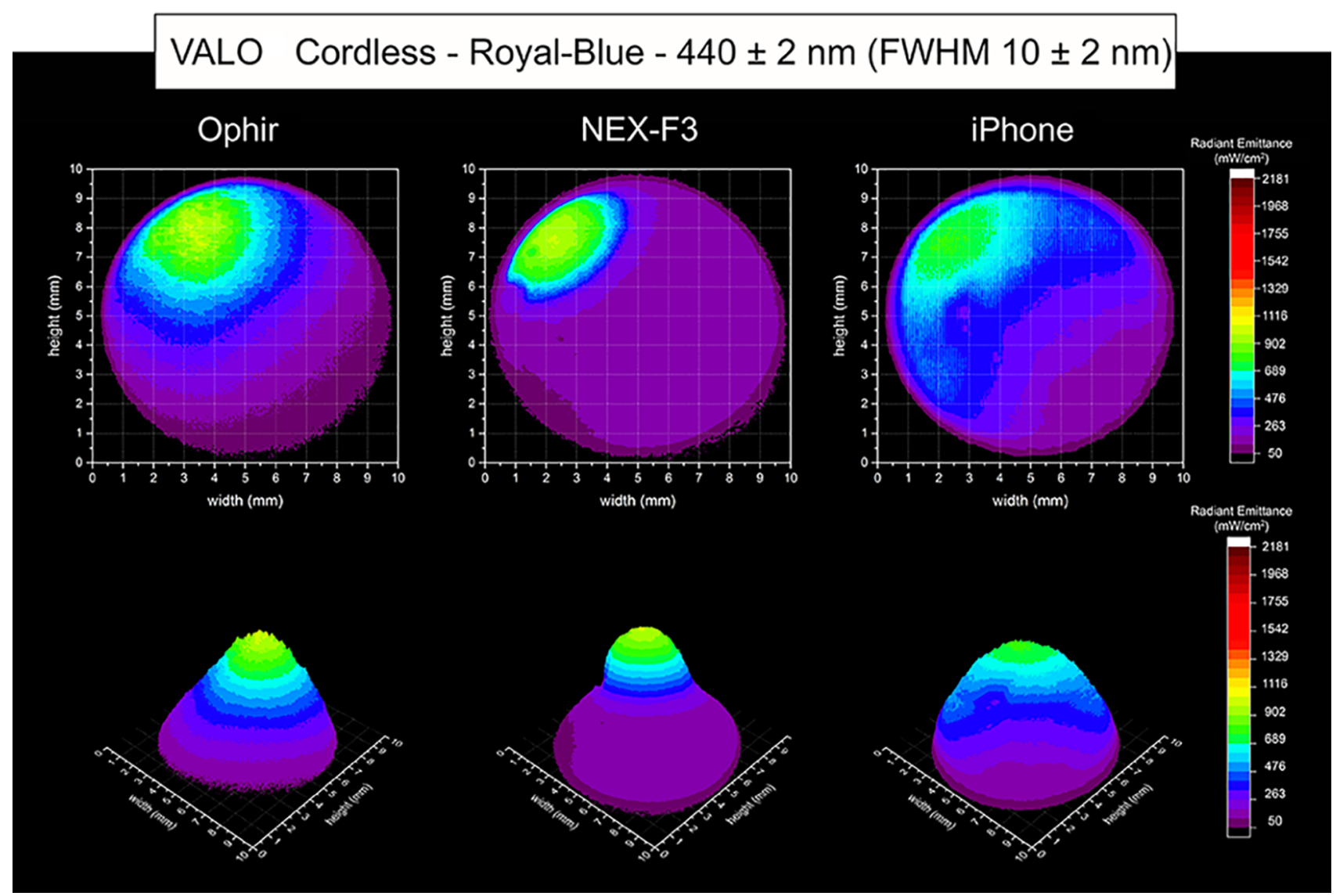

Fig. 8 Using the Ophir, NEX-F3 and iPhone cameras: 2D and 3D beam profile images of the VALO Cordless with the $440 \pm 2 \mathrm{~nm}$, full-width at half-maximum $10 \pm 2 \mathrm{~nm}$ bandpass filter showing the radiant emittance distribution.

However, recent advances in CMOS technology have steadily reduced the CMOS noise. As shown in - Table 1, the dynamic range of the Ophir camera $(64 \mathrm{~dB})$ is higher than the NEX-F3 (49 dB) and iPhone (42 dB) cameras. The dynamic range is the range that a sensor can detect from the lowest light intensity to the higher light intensity. That means that the Ophir camera can detect differences in a wider range than the NEX-F3 and iPhone camera. It is known that a camera with a higher dynamic range would have a lower signal-to-noise ratio and, as a consequence, a higher accuracy to detected differences in the power of the light beam. ${ }^{19,23,24}$ When considering the dynamic range of each camera in a minimum detection threshold of $50 \mathrm{~mW} / \mathrm{cm}^{2}$ for the characterization of a dental LCU, the Ophir camera can detect differences without pixel saturation up to $79,244.5 \mathrm{~mW} / \mathrm{cm}^{2}$ with an accuracy of $0.6 \mathrm{~mW} / \mathrm{cm}^{2}$. While, the NEX-F3 can detect differences without pixel saturation up to $14,091.9 \mathrm{~mW} / \mathrm{cm}^{2}$ with an accuracy of $3.5 \mathrm{~mW} / \mathrm{cm}^{2}$, and the iPhone can detect differences without pixel saturation up to $6,294.6 \mathrm{~mW} / \mathrm{cm}^{2}$, with an accuracy of $3.5 \mathrm{~mW} / \mathrm{cm}^{2}$. Indeed, the Ophir camera is by far better than the NEX-F3 and iPhone cameras. However, a recent study ${ }^{4}$ has shown that the maximum irradiance of the majority dental LCUs might have a range from 1,508 to $5,950 \mathrm{~mW} / \mathrm{cm}^{2}$, which means that both NEX-F3 and iPhone are still capable of performing the light collection without sensor oversaturation. However, some exceptions would be expected, such as the use of the iPhone camera for the beam profile characterization of the S.P.E.C. 3 (Coltene/Whaledent AG, Altstätten, Switzerland) that had a maximum irradiance of $8,325 \mathrm{~mW} / \mathrm{cm}^{2}{ }^{4}$ Thus, when choosing a low-budget camera to do a beam profile of a dental LCU, look for a CCD or a CMOS camera that has a dynamic range higher than $41 \mathrm{~dB}$.

Regarding the image resolution, the NEX-F3 and iPhone cameras have better resolution with more megapixels and smaller pixel sizes, which means that in terms of spatial distribution, those cameras would have better precision than the Ophir camera. However, these results do not rule out the influence of other factors such as the subpixel layout, as shown in -Table 1. The subpixel layout defines if a silicon-based camera sensor can detect light signals in grayscale or color by means of pixel intensities in different color channels (red, green, and blue [RGB]). In general, all camera sensors are currently monochrome, the photodiodes sensors that accept the photons and convert them into electrons, and so forth, all of them only deliver a level of gray as the sort of quantitative pixel intensity information. ${ }^{21}$ However, if filters are placed in front of them, levels of gray of a blue, green, or red channels can be detected. The mirrorless and smartphone cameras are designed to capture images with color depths; thus, necessarily, a microfilter in a Bayer array pattern with RGB in a 1:2:1 proportion is placed in front of the sensors. ${ }^{21}$ Considering that the wavelength range of the 


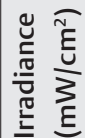

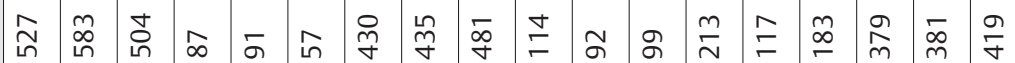

I

岇

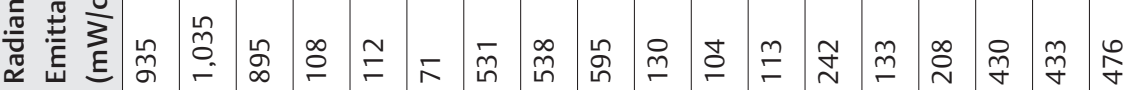

量离

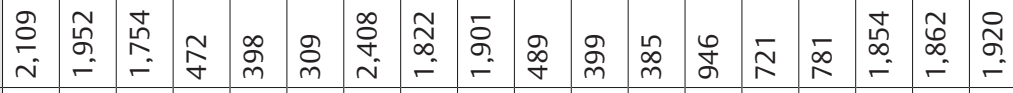

至

突 $\widetilde{\tilde{E}}$

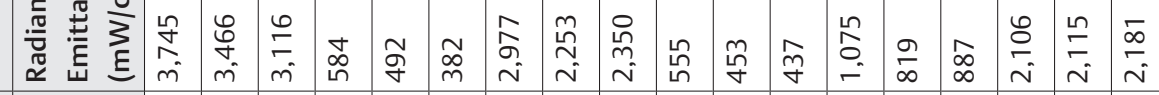

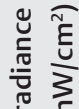

焉

至

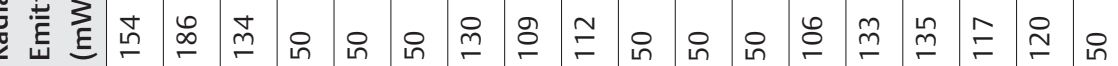

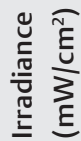

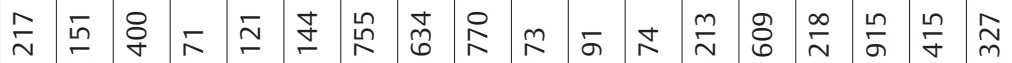

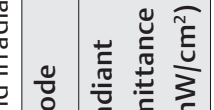

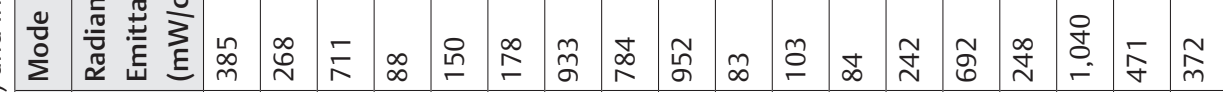

政

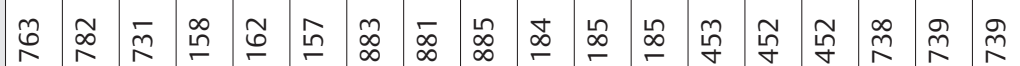

离

突

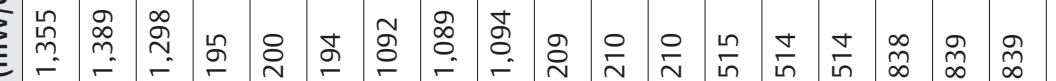

त्ञ

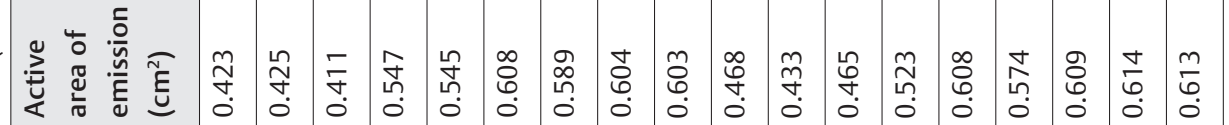

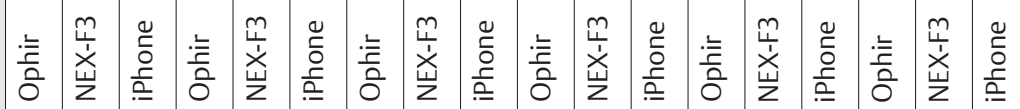
茪

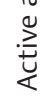

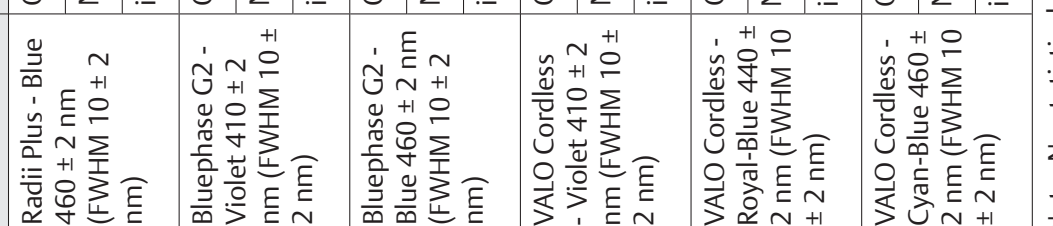




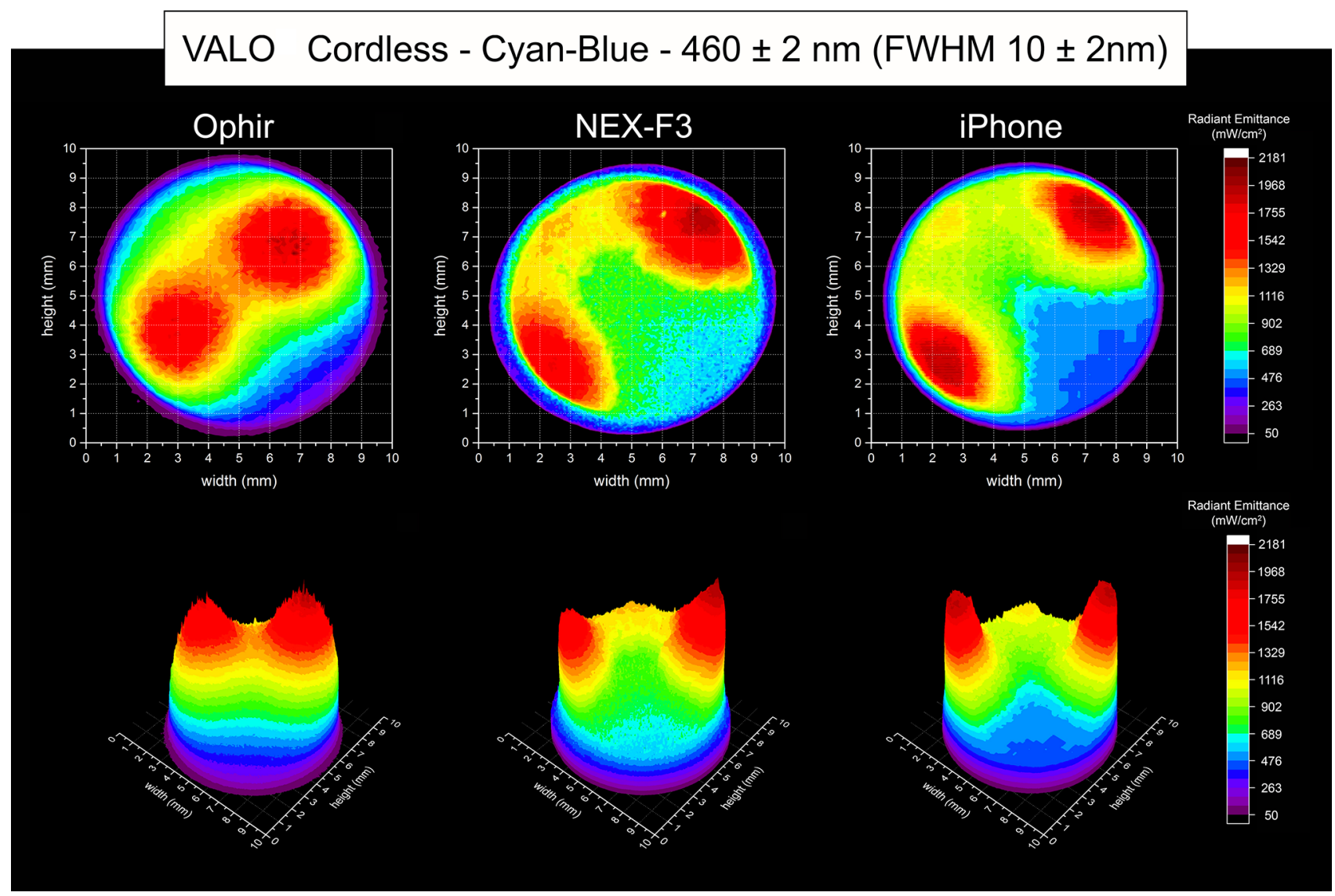

Fig. 9 Using the Ophir, NEX-F3 and iPhone cameras: 2D and 3D beam profile images of the VALO Cordless with the $460 \pm 2 \mathrm{~nm}$, full-width at half-maximum $10 \pm 2 \mathrm{~nm}$ bandpass filter showing the radiant emittance distribution.

Table 4 Correlation of the images using the NEX-F3 and iPhone cameras versus the Ophir camera

\begin{tabular}{|c|c|c|c|c|c|c|c|}
\hline \multirow[t]{2}{*}{ Light curing unit } & \multirow[t]{2}{*}{ Wavelength } & \multicolumn{3}{|l|}{ NEX-F3 } & \multicolumn{3}{|l|}{ iPhone } \\
\hline & & Pearson's r & $\begin{array}{l}\text { Adjusted } \\
\text { R-square }\end{array}$ & $p$-Value & Pearson's r & $\begin{array}{l}\text { Adjusted } \\
\text { R-square }\end{array}$ & $p$-Value \\
\hline Radii Plus & Blue & 0.92 & 0.85 & 0.0001 & 0.89 & 0.79 & 0.0001 \\
\hline \multirow[t]{2}{*}{ Bluephase G2 } & Violet & 0.94 & 0.89 & 0.0001 & 0.88 & 0.78 & 0.0001 \\
\hline & Blue & 0.92 & 0.84 & 0.0001 & 0.85 & 0.72 & 0.0001 \\
\hline \multirow[t]{3}{*}{ VALO Cordless } & Violet & 0.93 & 0.87 & 0.0001 & 0.95 & 0.89 & 0.0001 \\
\hline & Royal-Blue & 0.86 & 0.74 & 0.0001 & 0.84 & 0.71 & 0.0001 \\
\hline & Cyan-Blue & 0.89 & 0.79 & 0.0001 & 0.86 & 0.73 & 0.0001 \\
\hline
\end{tabular}

dental LCUs is within the blue channel of the Bayer array, it is almost certain that three quarters of the total pixels captured by the sensor are lost. As an example, the images obtained with the NEX-F3 and iPhone had higher resolution and more megapixels than the Ophir camera. However, it does not mean that after transforming the color image (color-chrome) obtained using the NEX-F3 and iPhone into a black and white image (monochrome), the transformed monochrome images would have better resolution than the original monochrome images obtained using the Ophir camera. The fact is that when the color-chrome images are transformed into a monochrome image, the color-coded pixels (red, green, or blue) are interpolated and reduced by four. This interpolation reduces the image resolution by estimating the pixel intensity from surrounding pixel intensity known values. As a consequence, making the image acquisition with larger pixels and more noise. Thus, it is suggested that when using color-chrome mirrorless or smartphone cameras for beam profile purposes that the camera should have at least $2,560 \times 1,920$ pixels ( 5.0 megapixel) of resolution to get similar results as the standard Ophir cameras.

When a photon from a dental LCU reaches the detector of a beam profile camera, its energy is converted from an electron-volt analog signal into a digital signal, as fully explained in many previous studies.22 Also, as mentioned before, the dynamic range is the range that a sensor can 
detect from the lowest light intensity to the highest light intensity. However, from the lowest to the highest pixel value, there is a continuous variable that could have an infinite number of possible values. Thus, the relation between the lowest to the highest signal detected by the sensor, associated with the amount of data stored digitally, defines the bit depth of the beam profile images. ${ }^{23}$ The bit depth is the number of intervals, or steps, between the minimum and the maximum pixel value, which is essential to differentiate the threshold between the value of two different pixels (the pixel value, for the beam profile images represents the irradiance $\left.\left(\mathrm{mW} / \mathrm{cm}^{2}\right)\right)$. Digital cameras usually have 8-bit $\left(2^{8}=256\right), 10$-bit $\left(2^{10}=1,024\right), 12$-bit $\left(2^{12}=4,096\right)$, or 16 -bit $\left(2^{16}=65,536\right) .{ }^{19}$ In terms of beam profile analysis, if a camera sensor has 8-bit, there is 256 levels for the pixel value; this means that if a dental LCU with a maximum irradiance of $1,000 \mathrm{~mW} / \mathrm{cm}^{2}$ is being evaluated, the camera can detect differences in irradiance of $3.9 \mathrm{~mW} / \mathrm{cm}^{2}$. Still, it is important to mention that if images are captured in JPEG format, the bit depth is limited to 8-bit, which gives 256 levels of gray and as consequence, 256 levels of irradiance, regardless of the bit depth of your camera sensor. ${ }^{24}$ However, images captured in RAW/DNG format can be anywhere from 10- to 16-bit, with the latter giving 65,536 levels of grays, meaning that it has a lot more accuracy in detect differences between pixels and when those images are converted to a TIFF file, there is no compression or loose of data. Thus, for the use of mirrorless or smartphone cameras as a beam profiling analysis system, the camera RAW file should be accessible because besides shooting the images without any digital processing, the images have more information (bit depth) about the dental LCU analyzed.

One of the main obstacles in beam profile analysis is to calibrate the images properly. The reason that occurs is because the absolute power fed to the camera is relative to the total power of the light beam. ${ }^{13,22}$ Therefore, it is essential to have an accurate method to collect the total power of the dental LCU. Otherwise, the irradiance distribution of the beam profile images would not be consistent. Several methods have been reported in the literature to collect the power $(\mathrm{mW})$ and the spectral irradiance $\left(\mathrm{mW} / \mathrm{cm}^{2} / \mathrm{nm}\right)$ of LCUs. ${ }^{4,12,13}$ It is almost certain that the method that uses an integrated sphere coupled to a spectrometer is the most reliable and precise of all methods. ${ }^{4}$ However, alternatives to that are also valid, but a note of caution is due here because each of these methods has their limitations..$^{25,26}$ In this study, a portable-spectrometer with a $16-\mathrm{mm}$ in diameter area of the collection was used to collect the spectral power $(\mathrm{mW} / \mathrm{nm})$ and the spectral irradiance $\left(\mathrm{mW} / \mathrm{cm}^{2} / \mathrm{nm}\right)$ of the dental LCUs from the entire light tip. It seems possible that other methods using a spectrometer coupled with a cosine corrector (MARC Resin Calibrator, BlueLight Analytics) might work as well, but it is important to mention that the power collected should be calibrated accordingly to the size of the cosine corrector (usually $3.9 \mathrm{~mm}$ in diameter) and not accordingly to the size of the light tip. In addition, cameras do not have uniform wavelength absorption. ${ }^{27}$ Therefore, they would have a different calibration factor for every wavelength of the light source that is used, and the attempt to calibrate the camera as a function of wavelength by using bandpass filters is the best way to get the best correlation among cameras. ${ }^{27}$ In this study, the intended purpose was to separate the royal- and cyan-blue spectrum, so a bandpass filter with a FWHM $10 \pm$ $2 \mathrm{~nm}$ was used. However, for more information regarding the use of bandpass filters with FWHM of $40 \mathrm{~nm}$ that covers the royal- and cyan-blue spectrum together, refer to the supporting information provided in this article.

There is a strong correlation between the dental LCU beam profile, and the physical and chemical properties of resin-based materials have been reported in the literature. ${ }^{6,7,28,29}$ The results of the present study broadly support the work of other studies that the beam profile of the dental LCU are notably different. ${ }^{4,7}$ There are many LCUs available in the market with a considerable variation in the number of LED chips, light tip sizes, and so many other aspects. All these variations can affect the beam profile of dental LCU, and it is essential to measure the light beam profile in any application if the energy distribution affects the performance of the dental LCU for its intended purpose.

The beam profile characterization of the Radii Plus, which represents a monowave LED LCU, showed big discrepancies between the radiant emittance at the center of the light tip and the irradiance in areas on the boundary of the light tip, regardless the camera used for beam profile analysis. The Radii Plus has a light tip with an external dimension of $1.2 \mathrm{~cm}$ in diameter. However, the inner exit window of the microlens has only $0.75 \mathrm{~cm}$ in diameter, resulting in an area of emission $0.442 \mathrm{~cm}^{2}$. Thus, Radii Plus had an average radiant emittance of approximately $1,378 \mathrm{~mW} / \mathrm{cm}^{2}$. But there were areas of higher radiant emittance at the center of the light tip with approximately $3,700 \mathrm{~mW} / \mathrm{cm}^{2}$, and areas of low radiant emittance at the periphery of the light tip with $160 \mathrm{~mW} / \mathrm{cm}^{2}$. One possible reason for these findings is probably due to the Radii Plus LCU design. The Radii Plus consist of four high-power LED chips at the center of the light tip associated to a plastic microlens that does not have much optical capability of mixing and collimate the light beam throughout the light tip.

The Bluephase G2 is a multiwave LED LCU with two peaks of emission. The beam profile showed differences in the radiant emittance and in the spectral radiant emittance regardless of the camera used for the analysis. The Bluephase G2 has a fiber optic light tip with an incoherent bundle and an external dimension of $1.0 \mathrm{~cm}$ in diameter. However, the active area of emission has $0.92 \mathrm{~cm}$ in diameter, resulting in an area of emission $0.635 \mathrm{~cm}^{2}$. The Bluephase $\mathrm{G} 2$ had an average radiant emittance of approximately $1,305 \mathrm{~mW} / \mathrm{cm}^{2}$. However, for the violet wavelength range, there were areas of higher radiant emittance $\left(\sim 555 \mathrm{~mW} / \mathrm{cm}^{2}\right)$ at the left part of the light tip and areas of low radiant emittance $\left(\sim 50 \mathrm{~mW} / \mathrm{cm}^{2}\right)$ on the bottom left of the light tip. For the blue wavelength range, there were areas of higher radiant emittance $\left(\sim 3,000 \mathrm{~mW} / \mathrm{cm}^{2}\right)$ at the top part of the light tip, and areas of low radiant emittance $\left(\sim 130 \mathrm{~mW} / \mathrm{cm}^{2}\right)$ on the bottom left of the light tip. These findings are also probably related to the Bluephase G2 design because the Bluephase G2 has four high-power LED chips 
emitting different wavelengths, three LED chips emitting blue light, and one LED chip emitting violet light). All four LED chips are located at the center of the body of the LCU associated with diffusive reflectors that orientate the light beam toward the boundary of the light tip. Plus, the coherent light tip bundle from the Bluephase G2 does not have any optical capability of mixing and collimate the light beam throughout the light tip.

The beam profile characterization of the VALO Cordless also showed differences in the irradiance and in the spectral irradiance regardless of the camera used for beam profile analysis. The VALO Cordless is a multiwave LED LCU with three different peaks of emission, two within the blue spectrum (at 440 [cyan-blue] and $460 \mathrm{~nm}$ [royal-blue]) and one within the violet spectrum (at $405 \mathrm{~nm}$ ). The VALO Cordless has a quartz microlens with an external dimension of $1.2 \mathrm{~cm}$ in diameter. However, the active area of emission of the VALO Cordless has $0.96 \mathrm{~cm}$ in diameter, resulting in an area of emission $0.691 \mathrm{~cm}^{2}$. The VALO Cordless had an average radiant emittance of approximately $1,066 \mathrm{~mW} / \mathrm{cm}^{2}$. However, for the violet wavelength range, there were areas of higher radiant emittance $\left(\sim 210 \mathrm{~mW} / \mathrm{cm}^{2}\right)$ on the bottom left part of the light tip, and low radiant emittance $\left(\sim 50 \mathrm{~mW} / \mathrm{cm}^{2}\right)$ on the top left of the light tip. For the royal-blue wavelength range, there were areas of higher radiant emittance $\left(\sim 1,075 \mathrm{~mW} / \mathrm{cm}^{2}\right)$ at the top part of the light tip, and low radiant emittance $\left(\sim 106 \mathrm{~mW} / \mathrm{cm}^{2}\right)$ on the bottom of the light tip. For the royal-blue wavelength range, there were two areas of higher radiant emittance $\left(\sim 2,100 \mathrm{~mW} / \mathrm{cm}^{2}\right)$, one at the top right and another on the bottom left part of the light tip, and areas of low radiant emittance $\left(\sim 120 \mathrm{~mW} / \mathrm{cm}^{2}\right)$ on the bottom right of the light tip. These findings are also probably related to the VALO Cordless design. The VALO Cordless has four high-power LED chips emitting different wavelengths, two LED chips emitting royal-blue light, one LED chip emitting cyan-blue light, and one LED chip emitting violet light. All LED chips are located at the center of the light tip associated with a quartz microlens that does mix the light beam throughout the light tip, but not completely.

In summary, a slight difference was noted between cameras, as showed and further explored in the histograms presented in the Supplementary Material (available in the online version). The results suggest that the mirrorless camera (NEX-F3) had a somewhat better performance than the smartphone camera (iPhone). However, it is important to point out that both the NEX-F3 and the iPhone cameras data showed a strong correlation with the data obtained using the Ophir camera. Thus, different cameras could be used in dentistry to perform the beam profile analysis of dental LCUs, since minimal requirements for the camera are respected. In a previous study, ${ }^{5}$ the authors attempted to use a digital single reflex camera and an iPad camera to analyze the light beam profile of different LCUs qualitatively. However, this study failed to address the statistical analysis of the images, and the conclusions are made on the assumption of visual comparison without any digital image correlation analysis. Besides that, the beam profiles images of the inactive light guide tips do not coincide with the real intensity distribution in all cases. With the referenced study based on imaging comparison in pseudo color, the methods reported do not provide any data reproducibility.

The findings in the present study have significant implications for understanding how imaging sensors' technology and how to adapt to dental LCU analysis. The methods reported here can contribute tremendously to further research projects and articles that intend to report the light-curing unit spectrum, irradiance, and beam profile. This study demonstrates and guides the reader on making a light beam profiler with different CCD/CMOS sensors. It can provide many authors with the opportunity to report the full characteristic of the LCUs used in their studies inexpensively and reproducibly. As state by Price et $\mathrm{al}^{2}$, "for improved interstudy reproducibility, reduced risk of premature failures, and ultimately better patient care, researchers and dentists need to know how to accurately characterize the electromagnetic radiation (light) they are delivering to the resins they are using." Overall, the present study strengthens the idea that it is very challenging to publish and validate any research paper that does not report the full characteristics of the studies' LCUs.

Still, a note of caution is due here since the number of LCUs and camera devices evaluated in this study is limited, and a more comprehensive study would include more groups. Questions remain unanswered at present, but this study directs further studies to pursue the development of camera gadgets and software, as well as the validation of these devices according to the ISO standards. Continued efforts are needed to make light beam profile and spectral analysis of the dental LCUs more accessible to dentists in the clinical practice.

\section{Conclusion}

This study demonstrated that mirrorless and smartphone cameras are able to perform beam profiling analysis of dental LCUs. The standard Ophir beam profile system presented the most accurate distribution, but the mirrorless and smartphone cameras presented a strong correlation in the irradiance distribution of the beam profile images. Specific requirements for the camera devices such as type of sensor, image bit depth, and image processing are important to achieve consistent results when using alternative methods for dental LCUs beam profiling.

\section{Funding}

This study was partially supported by Fundação de Ampáro a Pesquisa do Estado de São Paulo (grant 2016/06019-3, 2017/22195-9, 2016/05823-3, and 2017/22161-7).

\section{Conflict of Interest}

None declared.

\section{References}

1 Rueggeberg FA. State-of-the-art: dental photocuring-a review. Dent Mater 2011;27(1):39-52

2 Price RB, Ferracane JL, Shortall AC. Light-curing units: a review of what we need to know. J Dent Res 2015;94(9):1179-1186 
3 Price RB, Shortall AC, Palin WM. Contemporary issues in light curing. Oper Dent 2014;39(1):4-14

4 Shortall AC, Felix CJ, Watts DC. Robust spectrometer-based methods for characterizing radiant exitance of dental LED light curing units. Dent Mater 2015;31(4):339-350

5 Haenel T, Hausnerová B, Steinhaus J, Moeginger IB. Qualitative beam profiling of light curing units for resin based composites. Eur J Prosthodont Restor Dent 2016;24(4):197-202

6 Haenel T, Hausnerová B, Steinhaus J, Price RB, Sullivan B, Moeginger B. Effect of the irradiance distribution from light curing units on the local micro-hardness of the surface of dental resins. Dent Mater 2015;31(2):93-104

7 Shimokawa CAK, Turbino ML, Giannini M, Braga RR, Price RB. Effect of light curing units on the polymerization of bulk fill resin-based composites. Dent Mater 2018;34(8):1211-1221

8 Li X, Pongprueksa P, Van Meerbeek B, De Munck J. Curing profile of bulk-fill resin-based composites. J Dent 2015;43(6):664-672

9 de Oliveira DCRS, Rocha MG, Gatti A, Correr AB, Ferracane JL, Sinhoret MA. Effect of different photoinitiators and reducing agents on cure efficiency and color stability of resin-based composites using different LED wavelengths. J Dent 2015;43(12):1565-1572

10 Rocha MG, de Oliveira D, Correa IC, et al. Light-emitting diode beam profile and spectral output influence on the degree of conversion of bulk fill composites. Oper Dent 2017;42(4):418-427

11 Krames MR, Shchekin OB, Mueller-Mach R, et al. Status and future of high-power light-emitting diodes for solid-state lighting. J Displaing Technol 2007;3(2):160-175

12 Price RB, Labrie D, Rueggeberg FA, Felix CM. Irradiance differences in the violet $(405 \mathrm{~nm})$ and blue $(460 \mathrm{~nm}) \mathrm{spec}-$ tral ranges among dental light-curing units. J Esthet Restor Dent 2010;22(6):363-377

13 Price RB, Rueggeberg FA, Labrie D, Felix CM. Irradiance uniformity and distribution from dental light curing units. J Esthet Restor Dent 2010;22(2):86-101

14 Roulet J-F, Rocha MG, Shen C, Khudhair MM, de Oliveira DCRS. Beam profile characterization of a dental light curing unit using a spectrometer-based method. Stomatol Edu J 2018;5(2):84-91

15 Radii Plus SDI. Available at: https://www.sdi.com.au/ downloads/brochures/Radii_plus_Bro_EN.pdf

16 Vivadent I. Bluephase G2 and 20i. Available at: https:// pdf.medicalexpo.com/pdf/ivoclar-vivadent/bluephase-g220i/72878-195869.html. Accessed 2021
17 Ultradent. VALO Cordless. Available at: https://www.ultradent.com/products/categories/equipment/curing-lights/ valo-cordless. Accessed 2021

18 Schindelin J, Arganda-Carreras I, Frise E, et al. Fiji: an open-source platform for biological-image analysis. Nat Methods 2012;9(7):676-682

19 Battiato S, Castorina A, Mancuso M. High dynamic range imaging for digital still camera: an overview. J Electron Imaging 2003;12(3):459-469

20 Fossum ER. CMOS image sensors: Electronic camera-on-a-chip. IEEE Xplore 1997;44:1689-1698

21 Pei SC, Tam IK. Effective color interpolation in CCD color filter arrays using signal correlation. IEEE Trans Circuits Systems Video Technol 2003;13(6):503-513

22 Dickey FM, Holswade SC, Laser Beam Shaping: Theory and Techniques. 2nd edition. CRC Press Taylor \& Francis Group, United States; 2017

23 Duggin MJ, Jayne R, Loe R, Gregory J. Calibration requirements and the impact of bit depth for digital cameras used as imaging polarimeters. SPIE Proceedings 4133:Polarization Analysis, Measurement, and Remote Sensing III:179-90. Available at: https://spie.org/Publications/Proceedings/ Paper/10.1117/12.406625. Accessed 2000

24 Fridrich J, Goljan M, Du R. Lossless data embedding for all image formats SPIE 4675 . Security Watermark Multimedia Contents 2002;IV:572-583

25 Shimokawa C, Sullivan B, Turbino ML, Soares CJ, Price RB. Influence of emission spectrum and irradiance on light curing of resin-based composites. Oper Dent 2017;42(5):537-547

26 Harlow JE, Sullivan B, Shortall AC, Labrie D, Price RB. Characterizing the output settings of dental curing lights. J Dent 2016;44:20-26

27 Gaigalas AK, Wang L, He HJ, DeRose P. Procedures for wavelength calibration and spectral response correction of CCD array spectrometers. J Res Natl Inst Stand Technol 2009;114(4):215-228

28 Price RB, Labrie D, Rueggeberg FA, Sullivan B, Kostylev I, Fahey J. Correlation between the beam profile from a curing light and the microhardness of four resins. Dent Mater 2014;30(12):1345-1357

29 Sampaio CS, Atria PJ, Rueggeberg FA, et al. Effect of blue and violet light on polymerization shrinkage vectors of a CQ/TPO-containing composite. Dent Mater 2017;33(7):796-804 